\title{
HILGARDIA
}

A Journal of Agricultural Science Published by the California Agricultural Experiment Station

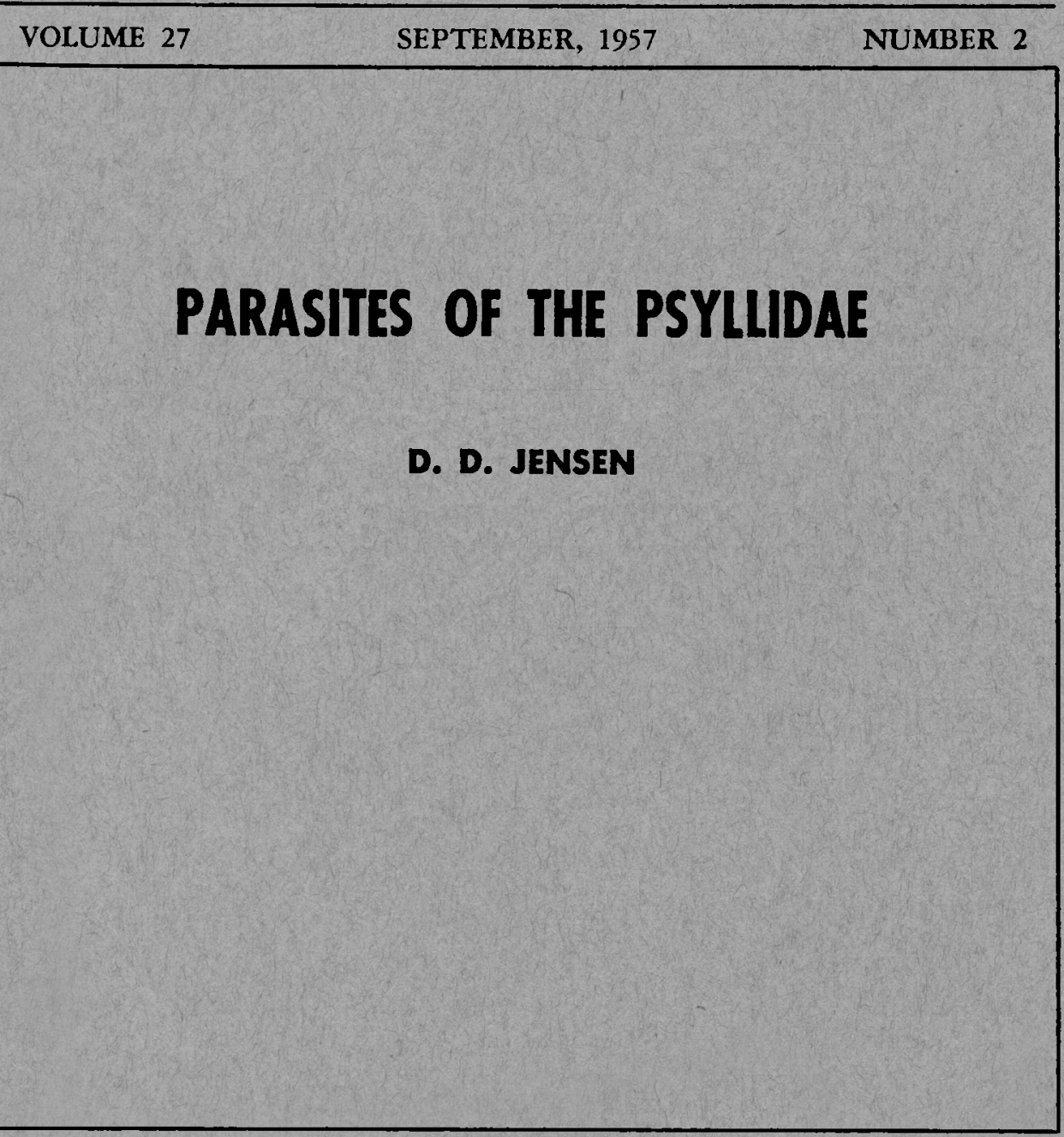

UNIVERSITY OF CALIFORNIA - BERKELEY, CALIFORNIA 
In the studies reported here, parasites of the Psyllidae were reared, identified, and stages of their development and their relations with their hosts were recorded. Parasites were reared from the nymphs of 11 genera and 34 species of Psyllidae. Results are combined with a summary of previously recorded observations on parasites of the Psyllidae. 


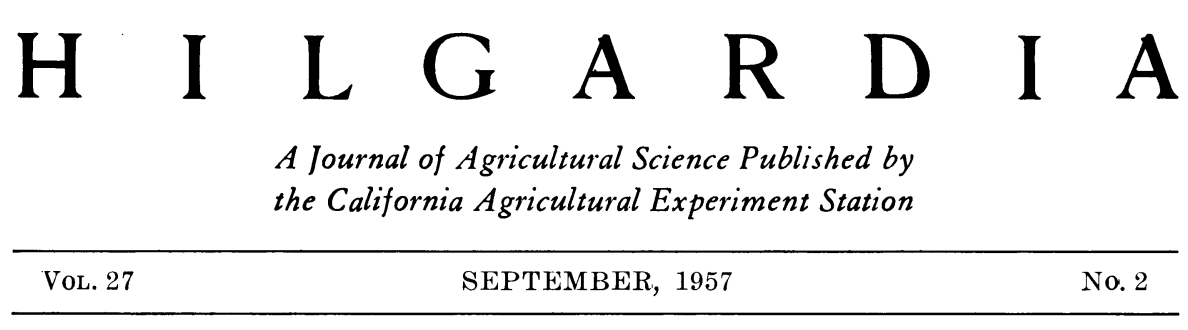

\section{PARASITES OF THE PSYLLIDAE}

\section{D. JENSEN ${ }^{2}$}

\section{INTRODUCTION}

In the course of a study of the Psyllidae, information relating to their parasites has been collected. This information consists both of observations made by the writer and of previously published racords. All of these have been combined and summarized in Tables I and 2. Table 1 lists the parasites of psyllids arranged according to order, superfamily, family, genus, and spe sies. Table 2 lists the psyllid species from which parasites have been rearer. In both tables the taxonomic groups are arranged in alphabetical rather than phylogenetic order. Geographical regions and literature references are given for the records cited. Except for the dipterous family Itonididae, all parasites are in the Hymenoptera.

Records have not been found of parasites attacking psyllid eggs. The nymphal stage of the psyllid is parasitized in all cases except those involving the gall midge genus Endopsylla which is endoparasitic in adult psyllids. The itonidid genera Lestodiplosis and Bremia are normally considered to be predatory in habit. Records of their attack on the Psyllidae are included here, however, because their relationship to the psyllid host is inadequately understood. Lal (1934) listed them as parasites of adult psyllids, but Rübsaamen (1901) has stated definitely that the psyllid "larvae" were the host stage attacked by Lestodiplosis liviae Rübs.

During the investigations reported here, a special effort has been made to rear as many of the Psyllidae as possible from the nymph to the adult stage to determine the nymphal characteristics of the various species. This is an indispensable basis for identifying the immature stages and for a more complete understanding of the relationships within the family. For example, certain species which have been considered closely related on the basis of adult characteristics are only distantly related if judged by those of the nymphs. Then too, because most parasites attack the nymphs rather than the adults, the nymphs must be identifiable if the host species is to be determined.

As part of the study, parasites were reared from the nymphs of 11 genera and 34 species of Psyllidae. Determinations to date indicate that 5 families

\footnotetext{
${ }^{1}$ Received for publication November 27, 1956.

${ }^{2}$ Associate Professor of Entomology and Associate Entomologist in the Experiment Station, Berkeley.
} 
and at least 8 genera and 15 species of parasitic Hymenoptera were represented in the reared material. Unless otherwise stated, all new records listed in the paper were based on collections and rearings made by the writer.

\section{HOST-PARASITE RELATIONSHIPS}

Habitat of psyllid nymphs. Among the psyllid species found parasitized in North America, the nymphs show wide variation in the degree to which they are exposed or concealed on the host plant. In several species the nymphs produce a waxy covering under which they feed. In most species, however, the nymphs are naked and feed on the relatively unmodified surface of the host plant. For some species of Arytaina most of the nymphal development takes place on the old wood of the host at or just below the ground level. In other species the feeding of the nymphs causes the infested leaves to curl so that the nymphs are sheltered inside. The hackberry psyllids (Pachypsylla spp.) cause the host plant to produce true galls of various types in which the nymphs develop.

At the present time there appears to be no relationship between the degree of parasitism and the apparent exposure and accessibility of the nymphs to parasite attack. In fact, the nymphs of Pachypsylla spp., which develop inside leaf and petiole galls, seem to have a higher percentage of parasitized nymphs than do most of the species in genera whose nymphs would appear to be more vulnerable to attack.

Development of parasites. All of the parasitized nymphs in this investigation had been parasitized in the field before they were collected. Thus specific information was not obtained on the time and mode of oviposition. In nearly all cases the nymphs were in the last or next-to-last instar before they showed evidence of being parasitized. Nymphs apparently remain active and show no conspicuous evidence of being parasitized for several days after the parasite has laid its egg. In one case a nymph of Arytaina essigi Jensen, confined alone in a vial with food, remained active for two weeks. Two adults of Prionomitus mitratus (Dalm.) subsequently emerged from this nymph, the second appearing three days after the first.

As the parasite larva develops, the activity of the nymph diminishes. Finally the nymph is represented only by its exoskeleton which assumes a swollen, parchment-like appearance. At this stage the parasite pupa can be seen through the nymphal integument.

Although most of the parasitic species develop within the body cavity of the nymph this is not the case for Tetrastichus triozae Burks and may not be true for some other species of Tetrastichus. Pletsch (1947) reported that in the laboratory Tetrastichus triozae females temporarily paralyzed the nymphs of Paratrioza cockerelli (Sulc) and then laid eggs on the ventral surface of the psyllid nymph's body-usually to one side of the mid-ventral line between the legs. He emphasized that, after hatching, the parasite larva remained outside the body wall of the nymph during its feeding and development, but cut its emergence hole through the dorsal surface of the nymphal shell.

In the present study Tetrastichus triozae has been reared from the nymphs of at least nine additional species of psyllids. In these cases the evidence also 
indicates that the parasite larva develops outside of the host body. In a number of instances the adult parasite was able to push the nymphal shell up enough to get out without cutting a hole through the integument of the nymph. In most cases, however, the nymphal shell becomes attached to the host plant by strands of an adhesive material, as previously observed by Pletsch (1947), who suggested that this resulted from the action of the parasite. This is an adaptation which insures protection for the parasite between the substratum and the nymphal shell during pupation. Parasites which develop and pupate within the body cavity of the psyllid nymph continue to have a measure of protection even if the parasitized nymph becomes dislodged from its host.

An unidentified species of Tetrastichus was collected while the larva was clearly attached to the outside venter of the nymph of Arytaina bicolor Jensen. Six days after collection, the larva pupated between the body of the nymph and the wall of the glass vial in which they were enclosed.

Only one adult parasite develops in a single nymph in nearly all cases. Two exceptions were encountered during this study. Two adult Prionomitus mitratus emerged from the same nymph of Arytaina essigi and three adults of Aphycus sp. were reared from a single nymph of Trioza minuta arizonae Aulmann.

Emergence of parasites. Typically, the parasite pupates in such a position that it lies on its back with its head in the abdomen of the psyllid nymph. When ready to emerge the parasite cuts a circular hole in the dorsal surface of the psyllid's abdomen. Occasionally emergence of Tetrastichus spp. was observed to be through a hole in the head or thorax of the nymph. The emergence of an adult of Prionomitus sp. was observed July 12, 1949. Parasitized nymphs of Arytaina robusta Crawford had been collected June 27. A round hole was chewed in the venter rather than in the dorsum of the nymph's abdomen because the parasitized nymph had been detached from its host and was lying on its back in the vial. The parasite attempted to emerge several times before it had cut the hole large enough to allow passage of the head and antennae. The material bitten off in making the hole was swallowed by the parasite.

Parasite distribution. Prionomitus mitratus is the only parasite species recorded from psyllids in both the New World and Europe. The first specimens of this species identified from America were among the psyllid parasites reared during this study. Of the 22 genera of hymenopterous parasites of the Psyllidae recorded in Table 1, seven genera are known only from North America, nine are known only from outside of the Americas and the distribution of six genera includes the New World and other regions.

Levels of Parasitism. Most of the psyllid species are not heavily parasitized as a general rule. At times, however, the proportion parasitized becomes relatively high. This has been determined most conclusively for certain of the economic species. In 1939 Pletsch (1947) found 23 per cent of the nymphs of Paratrioza cockerelli parasitized at Billings, Montana. No parasites were found among heavy psyllid infestations in other localities.

Eurytoma sp. was reported parasitizing 18.4 per cent of 365 nymphs of Pachypsylla celtidis-inteneris Mally (Smith and Taylor, 1953) in Kansas 
and Walton (1944) found the same hackberry psyllid parasitized to the extent of 31.6 per cent by Psyllaephagus pachypsyllae (Howard) in New York City.

Husain and Nath (1924) reported that in India as many as 95 per cent of the nymphs of the citrus pest, Diaphorina citri (Kuw.), were parasitized at some localities by several species of parasites. The most important parasite, and the only one identified, was Tetrastichus radiatus Waterston.

Host Relationships of Parasitic Genera and Species. The parasitic species reared from psyllid nymphs appear to be predominantly if not exclusively parasitic on the Psyllidae. An exception may be Tetrastichus sicarius Silvestri which is reported to have been reared from Coccus viridis Green in Africa and Mauritius as well as from the "Citrus Psylla," presumably Trioza citri Laing.

Asaphes vulgaris Walker (Pteromalidae) has been listed in the literature as emerging from aphids in several genera, aphidiids, and Curculionidae as well as from the apple psylla, Psylla mali (Schmidb.). However, these records cannot be interpreted literally because as Lal (1934) indicated, Asaphes vulgaris has been shown to be a hyperparasite.

The host specificity of some of the parasitic Hymenoptera attacking the Psyllidae is difficult to evaluate because in many cases only one record of parasitism has been published for some of the parasite species. Psyllid parasites in the genera Torymus (Callimome) and Eurytoma have all been reared from the gall-forming psyllids of the genus Pachypsylla. Three species of Alloxysta were reared from three different species of psyllids but all three were in genus Euphyllura.

In contrast to the above evidence are rearing records which indicate that other parasites show relatively little selectivity among the psyllids. The encyrtid Prionomitus mitratus is now known to parasitize 17 species of psyllids in 4 genera. If the specimens now provisionally labelled as Prionomitus sp. also prove to be $P$. mitratus, as seems probable, the total will increase to at least 21 species. Tetratichus triozae is recorded from 12 species in 7 genera.

Three different genera of parasites were reared from the nymphs of a single psyllid species collected at the same time and place.

The taxonomic position of parasites reared from psyllid nymphs is given below with a brief discussion of the general host relationships of the genera. The categories have been arranged in alphabetical rather than in phylogenetic order. The host groups listed are not to be construed as complete; they represent the more common hosts or indicate the diversity of hosts.

Except for the dipterous gall midge genera Endopsylla, Lestodiplosis, and Bremia, all the parasites recorded from the Psyllidae occur in the order Hymenoptera. Moreover, all the hymenopterous parasites attack only the nymphal stages of the Psyllidae.

\section{HYMENOPTEROUS PARASITES}

\section{Superfamily Chalcidoidea}

Encyrtidae. This family includes a large number of species most of which develop as internal parasites of the Homoptera, Hymenoptera, Lepidoptera,

${ }^{3}$ The names used in this paper follow Muesebeck, Krombein, and Townes (1951). 
Coleoptera, Diptera, Neuroptera, and less frequently in other insect orders and in ticks (Clausen, 1940).

A phycus. Host records for Aphycus refer almost exclusively to coccids or to chalcidoid parasites of coccids. Exceptions include two records from the Psyllidae. One of these concerns Aphycus (Metaphycus) psyllidis (Compere) which was found parasitizing the nymphs of the potato psyllid, Paratrioza cockerelli (Sulc) in California.

Cercobelus. The species $C$. jugaeus Walker has been recorded once as a parasite of the psyllid Psyllopsis fraxinicola (Förster) in Scotland. Other host records for the genus have not been found.

Cheiloneurus. The insect hosts of this genus are principally primary parasites of soft scales and other Homoptera. Two psyllid species have also been recorded as hosts, but it is probable that the Cheiloneurus were secondary parasites in these instances.

Encyrtus. The hosts of this genus include the Psyllidae, Diptera, Lepidoptera, and Coleoptera, in addition to the soft scales which are the most common hosts.

Metallon. This genus appears to be recorded only from the Psyllidae.

Mirocerus. This genus was described (Ashmead, 1904) for a parasite reared from a psyllid. The original publication lists the type species as "peyelae." However, Gahan and Peck (1946) point out that this was a typographical error because the type, in the U.S. National Museum, is plainly labeled "psyllae."

Prionomitus. This genus appears to be parasitic principally on psyllids although it has also been recorded from Lecanium. Among the Psyllidae, Prionomitus mitratus is recorded from at least 13 species in four genera.

Psyllaephagus. The 10 named species in this genus were all reared from the Psyllidae. In the present study, undescribed species of Psyllaephagus were reared from 10 species in seven genera of psyllids.

Psylledontus. Three parasite species in the genus Psylledontus have been reared from three different species of psyllids in Ceylon, Japan, and New York, respectively.

Psyllencyrtus. This monotypic genus was described in 1955 for a new parasite species reared from the psyllid, Syntomoza magna Kuwayama.

\section{EULOPHIDAE}

Aphelinus (Agonioneurus). Members of the genus Aphelinus are principally parasitic on aphids, but species have also been reported from Psyllidae, Coccidae, and even Coleophoridae.

Aprostocetus. This genus is predominantly parasitic on gall midges, but one species was reared from a psyllid and other records indicate Orthoptera, Lepidoptera, and Coleoptera may be hosts.

Pteroptrix. The only host record found for this genus listed the psyllid Rhinocola eucalypti Mask. in New Zealand.

Tetrastichus. The arthropod host list of the genus Tetrastichus is very extensive. The major groups listed by Burks (1943) as being parasitized by Tetrastichus are Orthoptera, Odonota, Thysanoptera, Homoptera, Neuroptera, Coleoptera, Lepidoptera, Diptera, Hymenoptera, and Araneae. At least eight species are now known to parasitize over 20 species of psyllids. Waterston (1922) thought the Tetrastichus species might be secondary rather than 
primary parasites. There can be no question, however, regarding the primary parasitic status of $T$. triozae.

\section{EUPELMIDAE}

Eupelmus. Certain species in this genus are normally primary parasites but may also develop as hyperparasites. Only one record is available indicating psyllids as hosts. Moser (1956) reported that Eupelmus sp. had been observed ovipositing in the galls of Pachypsylla celtidis-vesicula Riley in Ohio.

\section{EURYTOMIDAE}

Eurytoma. Like the genus Torymus, Eurytoma species are primarily parasitic on gall-forming insects such as the Cynipidae, some of the Diptera, Lepidoptera, and Coleoptera. It is of interest that the psyllids attacked by both genera (Torymus and Eurytoma) occur in the Pachypsylla which form galls on hackberry (Celtis spp).

\section{PTEROMALIDAE}

Amblymerus. One record was found of a species of Amblymerus being reared from psyllids. Other parasitic records report various Coleoptera, Lepidoptera, Hymenoptera, and Diptera as hosts.

Asaphes. Species of this genus have been recorded from aphids, psyllids (one record), mealybugs, parasitic Hymenoptera, Coleoptera, and Diptera. However, it is questionable whether A. vulgaris Walker actually is a primary parasite of psyllids because this species has been shown to be a hyperparasite (Lal, 1934).

Pachyneuron. Three species of Pachyneuron have been described as parasites of Psyllidae. Other recorded hosts of the genus have been reported among the aphids, coccids, Neuroptera, Hymenoptera, Coleoptera, Lepidoptera, and Diptera.

\section{THYSANIDAE}

Thysanus (Signiphora). Members of this genus have been recorded most frequently from Aleyrodidae, Coccidae, and Pseudococcidae, and in one instance each from Psyllidae and Aphididae. However, Clausen (1940) considers it probable that they are predominantly hyperparasitic in habit.

\section{TORYMIDAE}

Torymus (Callimome). This genus is parasitic principally on insects causing plant galls, such as the Cynipidae, but other host records exist among the Diptera, Lepidoptera, Coleoptera, and Homoptera. Two species attack psyllids of the genus Pachypsylla in North America. Both of these species of Psyllidae cause galls on hackberry (Celtis spp.)

\section{CYNIPIDAE}

\section{Superfamily Cynipoidea}

Alloxysta. Three species of Alloxysta have been recorded from the Psyllidae. Although one species is widely separated geographically from the other two (Eritrea and California, respectively), all three of the host species belong to the genus Euphyllura. Alloxysta has also been recorded from aphids as secondary braconid parasites. They may also be secondary parasites when reared from psyllids. 


\section{BRACONIDAE}

\section{Superfamily Ichneumonoidea}

Aphidius. Almost all of the known hosts of Aphidius spp. are aphids. In addition, there is a single record from the Psyllidae.

\section{CeRAPHRONIDAE}

\section{Superfamily Proctotrupoidea}

Lygocerus. This genus is considered by Clausen (1940) and Lal (1934) to be primarily hyperparasitic on mealybugs, aphids, psyllids, and perhaps other Homoptera. In Scotland Lal (1934) reared Lygocerus semiramosus Kieffer from parasitized nymphs of Psylla peregrina Först. However, he believed these to be secondary in the psyllid and primary in the encyrtid Prionomitus mitratus which was parasitizing 20 to 30 per cent of the nymphs.

\section{PLATYGASTERIDAE}

Platygaster. Species of Platygaster are characteristically parasitic on gall-producing insects, particularly the Itonididae. Other hosts include Lepidoptera, Coleoptera, Coccidae, and Psyllidae.

\section{DIPTEROUS PARASITES}

Itonididae. Although the great majority of gall midges are phytophagous, the family includes approximately 50 species whose larvae are known to be predaceous and three species in two genera whose larvae are endoparasites.

Barnes (1930 and 1954) has reviewed the zoophagous gall midges in detail. Approximately 40 species of midge larvae are predaceous on scale insects and a few species have been recorded as predators of the Aphididae, Tingidae, Aleyrodidae, and Psyllidae.

In contrast to the hymenopterous parasites of the Psyllidae which always attack the nymphs, the gall midges attack the psyllid adults and, in some cases, perhaps also nymphs.

Endopsylla. The genus includes endoparasites of adult psyllids and tingids. Endopsylla agilis de Meijere was reported by de Meijere (1907) to parasitize Psylla foersteri Flor on alder in Holland, with from one to four parasites being reared from a single psyllid. Bagnall and Harrison (1924)recorded this parasite from the same psyllid species in England. In Germany, Speyer (1929 and 1941) reported that 19 per cent of the adults of the apple sucker, Psylla mali (Schmidb.), were parasitized by an Endopsylla which was provisionally identified as $E$. agilis.

Lal (1934) studied the biology of an Endospylla species in Scotland which Barnes (1954) thinks may prove to be $E$. agilis. The eggs are laid on the forewings of the psyllid, hatch in 8 to 13 days, feed for 3 or 4 days as ectoparasites, and then burrow into the haemocoele of the host. He recorded the species as a parasite of Psylla melanoneura Foerst., $P$. pyricola Foerst., $P$. mali, and P. peregrina. Parasitization of the last species ranged between 20 and 40 per cent. The affected psyllids became swollen and sluggish but did not change color.

Lestodiplosis and Bremia. The larvae of these genera are predatory. Lestodiplosis liviae Rübs. was described from specimens which Rübsaamen (1901) reared from the galls of Livia juncorum Latr. in Germany. He stated that the gall midge larvae feed on the larvae of Livia juncorum. Kieffer 
(1901) reported an undetermined species of Lestodiplosis which "lives at the expense of Aphalara maculipennis Fr. Lw." and that Rübsaamen had observed a species of Bremia in the galls of Psyllopsis fraxini (L.).

TABLE 1

SUMMARY OF PARASITES REARED FROM PSYLLID NYMPHS*

\begin{tabular}{|c|c|c|c|}
\hline Parasite & Host species & Region & Reference \\
\hline \\
\hline \multicolumn{4}{|l|}{ SUPERFAMILY CHALCIDOIDEA } \\
\hline \multicolumn{4}{|l|}{ Encyrtidae: } \\
\hline \multicolumn{4}{|l|}{ Aphycus (Metaphycus) psyllidis } \\
\hline 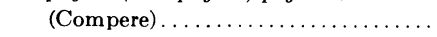 & Paratrioza cockerelli (Sulc) & California & Compere, 1943 \\
\hline Aphycus sp.. & Trioza minuta arizonae Aulmann & California & Present paper \\
\hline Cercobelus jugaeus Walker.............. & Psyllopsis fraxinicola Först. & Scotland & Lal, 1934 \\
\hline Cheiloneurus praenitens Waterston.. & Psyllid gall (?Trioza sp.) & Jamaica & Waterston, 1922 \\
\hline Cheiloneurus sp.............. & Arytaina amorphae (Mally) & California & Present paper \\
\hline Encyrtus pulvinatus Waterston. & Trioza citri Laing & Kenya & Waterston, 1922 \\
\hline Encyrtus triozae André. & Trioza centranthi Vallot & France & André, 1878 \\
\hline Encyrtus sp........... & Psylla pyricola Först. & Russia & Yakhontov, 1929 \\
\hline Metallon (Trechnites) psyllae Ruschka. & Psylla pyricola Först. & Germany & Ruschka, 1923 \\
\hline Mirocerus psyllae Ashm. & Psyllid & Ceylon & $\begin{array}{l}\text { Ashmead, } 1904 \\
\text { Gahan \& Peck, } \\
1946\end{array}$ \\
\hline Prionomitus mitratus (Dalm.). & Arytaina essigi Jensen & California & Present Paper \\
\hline Prionomitus mitratus (Dalm.). & Arytaina fuscip & Utah & Present paper \\
\hline Prionomitus mitratus (Dalm.). & Arytaina insolita Tut. & California & Present \\
\hline Prionomitus mitratus (Dalm.). & Arytaina minuta Crawf. & California & Present \\
\hline Prionomitus mitratus (Dalm.) & Arytaina "robusta" Crawf. & California & Present \\
\hline Prionomitus mitratus (Dalm.). & Pexopsylla cerc & $\mathrm{Ca}$ & Present \\
\hline Prionomitus mitratus (Dalm.). & Psylla america & & Presen \\
\hline Prionomitus & Psylla crato & and & Wille, \\
\hline Prionomitus mit & Psylla melanor & ind & Wille, 1950 \\
\hline Prionomitus mitratus & Psylla near media Tuthill & $\mathrm{Ca}$ & Present paper \\
\hline Prionomitus mit & Psylla pe & and & Lal, 1934 \\
\hline Prionomitus mitrat & Psylla $p$ & zerland & Wiıle, 1950 \\
\hline Prionomitus mitratus (D) & Psylla pyricola Först. & Switzerland & Wille, 1950 \\
\hline Prionomitus mitratus (Dalm & Psylla pyrisuga Först. & France & Ferrière, 1926 \\
\hline & & Switzerland & Brocher, 1926 \\
\hline & & & \\
\hline Prionomitus mitratus (Dalm & Psylla retamae Put. & Spain & Mercet, 1926 \\
\hline Prionomitus mitratus ( & Psylla ribesiae (Crawf.) & New Mexico & Jensen, 1956 \\
\hline Prionomitus mitratus (Dalm.) & Trioza beameri Tuthill & California & Present paper \\
\hline Prionomitus sp. & Arytaina bicolor Jensen & California & Present paper \\
\hline Prionomitus sp. & Arytaina "robusta" Crawf. & California & Present paper \\
\hline Prionomitus sp.. & Psylla americana Crawf. & California & Jensen, 1951 \\
\hline Prionomitus sp.. & Psylla breviata Patch & California & Jensen, 1951 \\
\hline & & & Pre \\
\hline Prio & Psylla & California & Present paper \\
\hline Prion & Psylla $m$ & Poland & Woroniecka, 1928 \\
\hline Prio & Psylla pyricola Först. & & Grandi, 1951 \\
\hline Prio & Psylla tenuata Jensen & California & Jensen, 1951 \\
\hline Prionomitus & Psylla sp. & Iran & Kiriukhin, 1946 \\
\hline $\begin{array}{l}\text { Psyllaephagus arbuticola Gahan \& } \\
\text { Waterston.............................. }\end{array}$ & Euphyllura arbuti Schw. & California & $\begin{array}{l}\text { Gahan \& Waters- } \\
\text { ton, } 1926\end{array}$ \\
\hline Psyllaephagus cellulatus Waterston & Rhinocola & Mesopotamia & Waterston, 1922 \\
\hline Psyllaephagus euphyllurae & Euphyllura olivina (Costa) & Italy, Sicily, & Masi, 1911; \\
\hline & & & Gahan \& \\
\hline Psyllaephagus femoralis Borell & Trioza alacris Flor & Italy & Borelli, 1920 \\
\hline Psyllaephagus iwayaensis Ishii. & Psyllid on Cinnamomum sp. & Japan & Ishii, 1928 \\
\hline Psyllaephagus metallicus (Girault). & "Bred out of Eucalyptus" & Australia & Girault, $1915 a$ \\
\hline Psyllaephagus pachypsyllae (Howard). & Pachypsylla celtidis-gemma Riley & Maryland & Howard, 1885 \\
\hline Psyllaephagus pachypsyllae (Howar & Pachypsylla venusia O.S. & Kansas & $\begin{array}{l}\text { Smith \& Taylor, } \\
1953\end{array}$ \\
\hline
\end{tabular}

* Except Endopsylla (Itonididae: Diptera) which were reared from psyllid adults. 
TABLE 1 (continued)

\begin{tabular}{|c|c|c|c|}
\hline Parasite & Host species & Region & Reference \\
\hline \multicolumn{4}{|l|}{ Encyrtidae-(Cont'd.) } \\
\hline Psyllaephagus pachypsyllae (Howard)... & Trioza beameri Tuthill & California & Present paper \\
\hline $\begin{array}{l}\text { Psyllaephagus sp. near pachypsyllae } \\
\quad(\text { Howard }) \ldots \ldots \ldots \ldots \ldots \ldots \ldots \ldots\end{array}$ & $\begin{array}{l}\text { Pachypsylla venusta O.S. or } P \text {. } \\
\text { celtidis-vesicula } \mathrm{Cr} \text {. }\end{array}$ & Idaho & Jensen, 1946 \\
\hline Psyllaephagus phytolymae Ferrière.... & Phytolyma lata Scott & Nigeria & $\begin{array}{l}\text { Ferrière, } 1931 \\
\text { Vosseler, } 1906\end{array}$ \\
\hline & & Gold Coast & Anon., 1940 \\
\hline Psyllaephagus solus (Howard). & Trioza magnoliae (Ashm.) & Florida & Howard, 1885 \\
\hline Psyllaephagus trioziphagus (Howard) & Trioza diospyri (Ashm.) & $\begin{array}{l}\text { Washington, } \\
\text { D.C.;Ohio }\end{array}$ & $\begin{array}{l}\text { Howard, 1885; } \\
\text { Caldwell, } 1938\end{array}$ \\
\hline Psyllaephagus sp... & Aphalara suaedae Crawf. & California & Present paper \\
\hline Psyllaephagus sp.. & Aphalaroida pithecolobia Crawf. & California & Present paper \\
\hline Psyllaephagus sp.. & Arytaina aculeata Crawf. & California & Present paper \\
\hline Psyllaephagus sp... & Arytaina amorphae (Mally) & California & Present paper \\
\hline Psyllaephagus sp... & Arytaina ceanothi Crawf. & California & Present paper \\
\hline Psyllaephagus sp.. & Arytaina "robusta" Crawf. & California & Present paper \\
\hline Psyllaephagus sp... & Euphyllura arbuti Schwarz & California & Present paper \\
\hline Psyllaephagus sp.... & Pexopsylla cercocarpi Jensen & California & Present paper \\
\hline Psyllaephagus sp................ & Psylla minuta Crawf.? & California & Present paper \\
\hline Psyllaephagus sp.............. & Psylla pyricola Först. & Italy & $\begin{array}{l}\text { Golfari, } 1937 \\
\text { Grandi, } 1951\end{array}$ \\
\hline Psyllaephagus sp... & Trioza bakeri Crawf. & California & Present paper \\
\hline Psylledontus insidiosus Crawford, . & Psylla pyricola Först. & $\begin{array}{l}\text { New York } \\
\text { Connecticut }\end{array}$ & $\begin{array}{l}\text { Crawford, 1911; } \\
\text { Anon., 1931 } \\
\text { Garman \& } \\
\text { Townsend,1941 }\end{array}$ \\
\hline Psylledontus secundus Girault & Gall-making psyllid & Ceylon & Girault, 1915 \\
\hline Psylledontus viridiscutellatus Ishii. & Psyllid on Elaeagnus sp. & Japan & Ishii, 1928 \\
\hline Psyllencyrtus syntomozae Tachikawa & Syntomoza magna Kuwayama & Japan & Tachikawa, 1955 \\
\hline (Undetermined) $\ldots \ldots \ldots \ldots$ & Trioza vitreoradiata (Mask.) & New Zealand & Carter, 1949 \\
\hline \multicolumn{4}{|l|}{ Eulophidae: } \\
\hline Aphelinus (Agon & Trioza centranthi Vallot & France & André, 1878 \\
\hline A prostocetus phytolymae Risbec... & Phytolyma lata var. fusca A libert & Gold Coast & $\begin{array}{l}\text { Anon. } 1940 \\
\text { Alibert, } 1947\end{array}$ \\
\hline & & Nigeria? & Risbec, 1947 \\
\hline A prostocetus roseveari Ferrière.... & Phytolyma lata Scott & Nigeria & Ferrière, 1931 \\
\hline ? Chrysocharoideus sp....... & Pachypsylla celtidis-vesicula Crawf. & Ohio & Moser, 1956 \\
\hline & & New York & Moser, 1956 \\
\hline Pteroptrix maskelli Ashm. & Rhinocola eucalypti Mask. & New Zealand & Gourlay, 1930 \\
\hline Tetrastichus clypeatus Gahan & Dynopsylla robusta Crawf. & Philippines & Gahan, 1925 \\
\hline Tetrastichus dryi Waterston. . & Trioza citri Laing & Kenya & Waterston, 1922 \\
\hline Tetrastichus dyrus Burks... & Trioza sp. & Montana & Burks, 1943 \\
\hline Tetrastichus gelastus Burks. & Trioza sp. & Florida & Burks, 1943 \\
\hline Tetrastichus obscuratus André.. & Trioza centranthi Vallot & France & André, 1878 \\
\hline & Trioza urticae L. & Italy & $\begin{array}{l}\text { Grandi, } 1953 \\
\text { Zangheri, } 1954\end{array}$ \\
\hline Tetrastichus radiatus Waterston. & Diaphorina citri (Kuw.) & India & $\begin{array}{l}\text { Husain \& Nath, } \\
1927\end{array}$ \\
\hline Tetrastichus sicarius Silvestr & "Citrus Psylla" & Kenya & Waterston, 1922 \\
\hline Tetrastichus triozae Burks... & Arytaina minuta Crawf. & California & Present paper \\
\hline Tetrastichus triozae Burks... & Calophya californica Schw. & California & Present paper \\
\hline Tetrastichus triozae Burks... & Calophya nigrella Jensen & California & Present paper \\
\hline Tetrastichus triozae Burks... & ? Calophya nigripennis Riley & Kansas & Burks, 1943 \\
\hline Tetrastichus triozae Burks. & Calophya triozomima Schw. & California & Present paper \\
\hline T'etrastichus triozae Burks. & Euphalerus vermiculosus Crawf. & California & Present paper \\
\hline Tetrastichus triozae Burks.. & Pexopsylla cercocarpi Jensen & California & Present paper \\
\hline
\end{tabular}

$\dagger$ Bred from the same nymph that gave rise to Encyrtus triozae and thought by Andre to be a hyperparasite. 
TABLE 1 (continued)

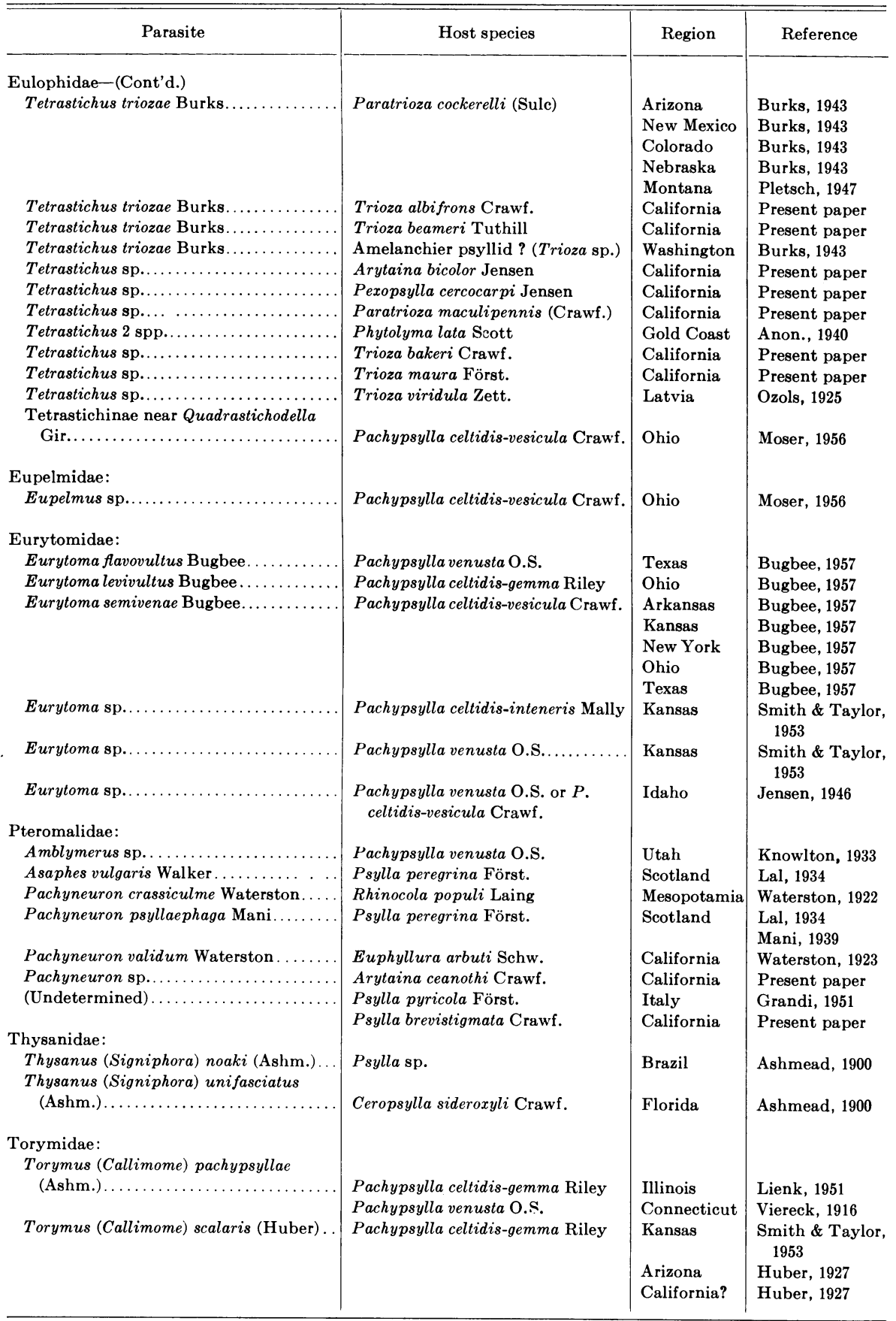


TABLE 1 (continued)

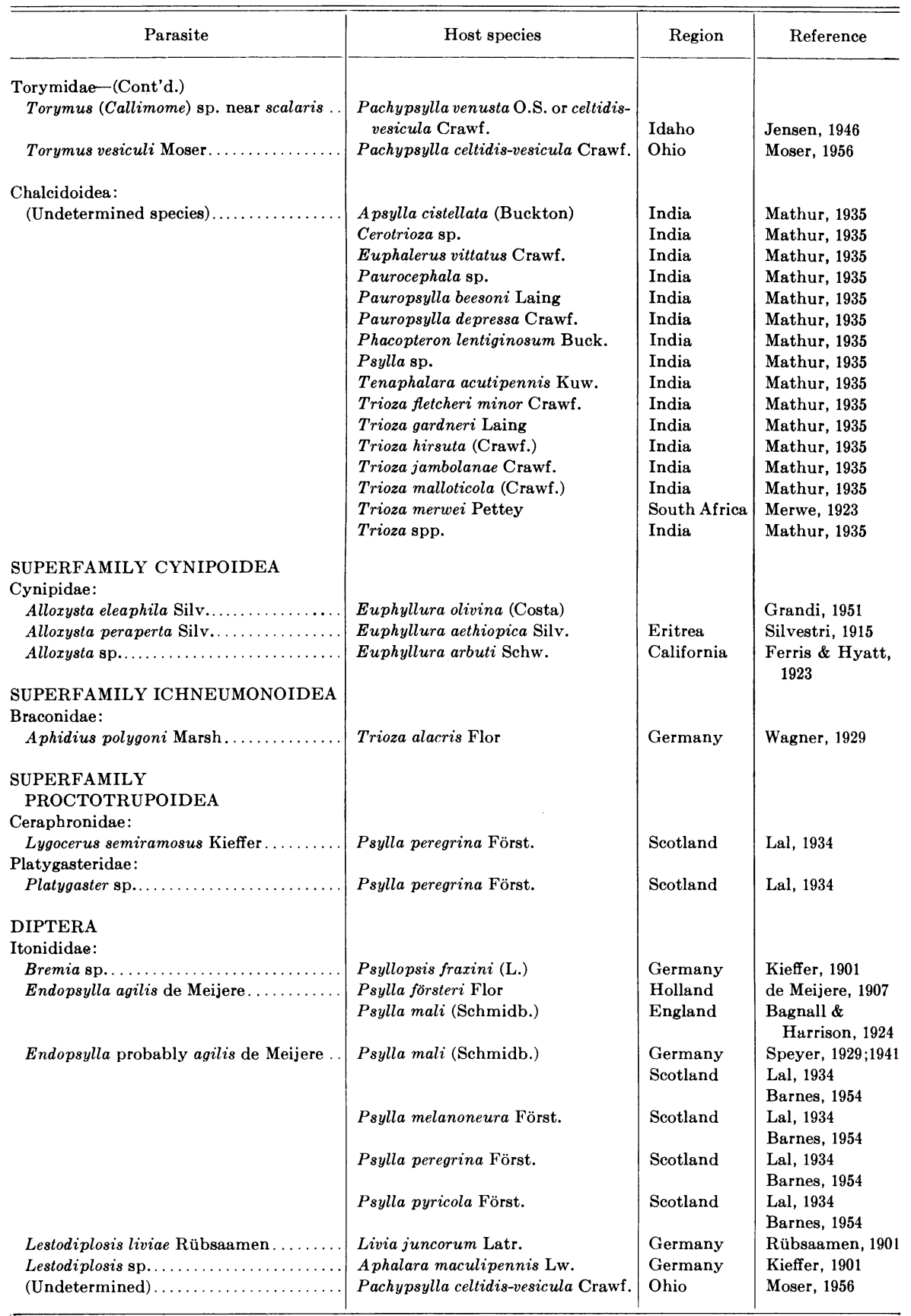


TABLE 2

PSYLLID SPECIES FROM WHICH PARASITES HAVE BEEN REARED

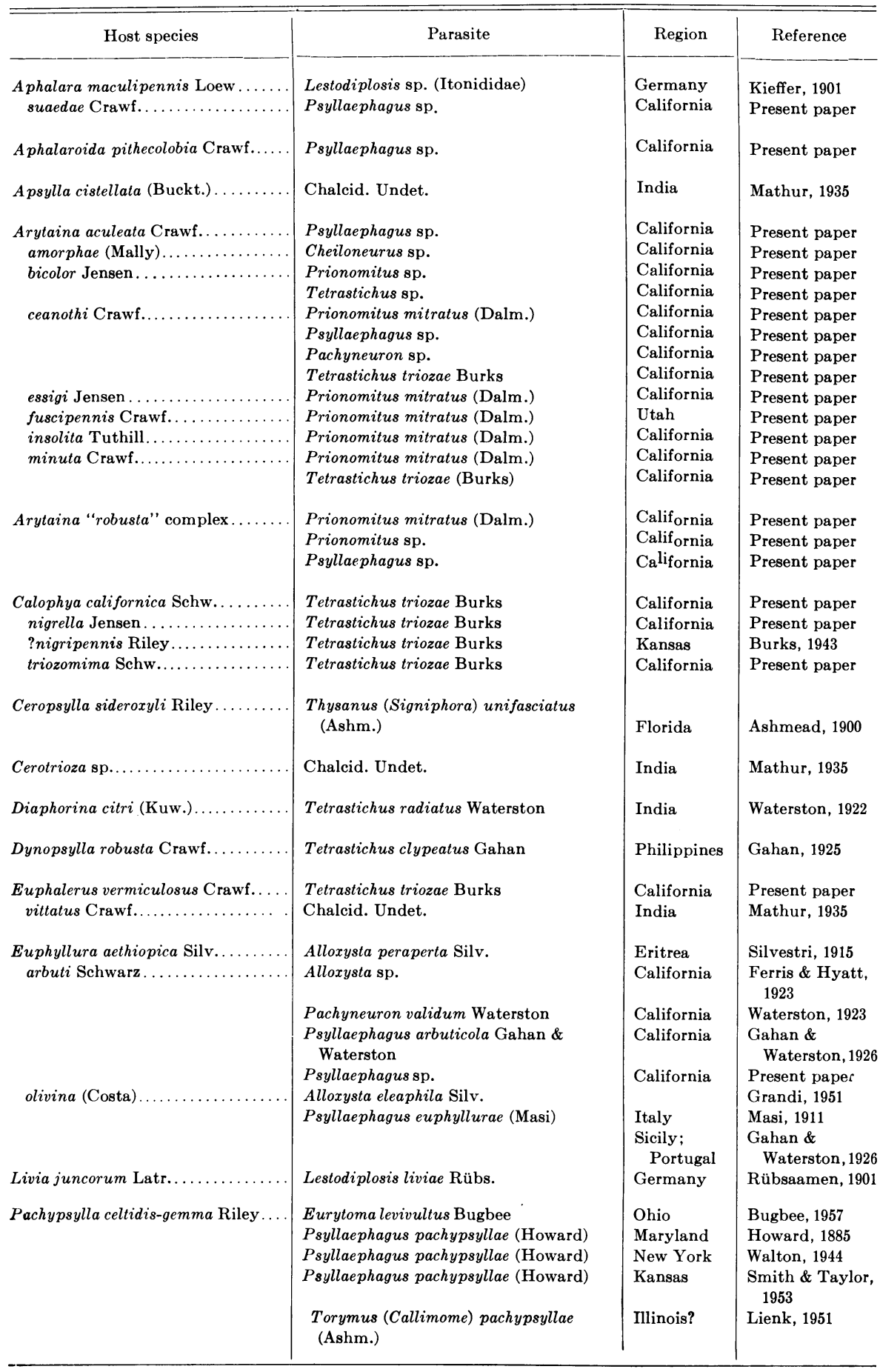


TABLE 2 (continued)

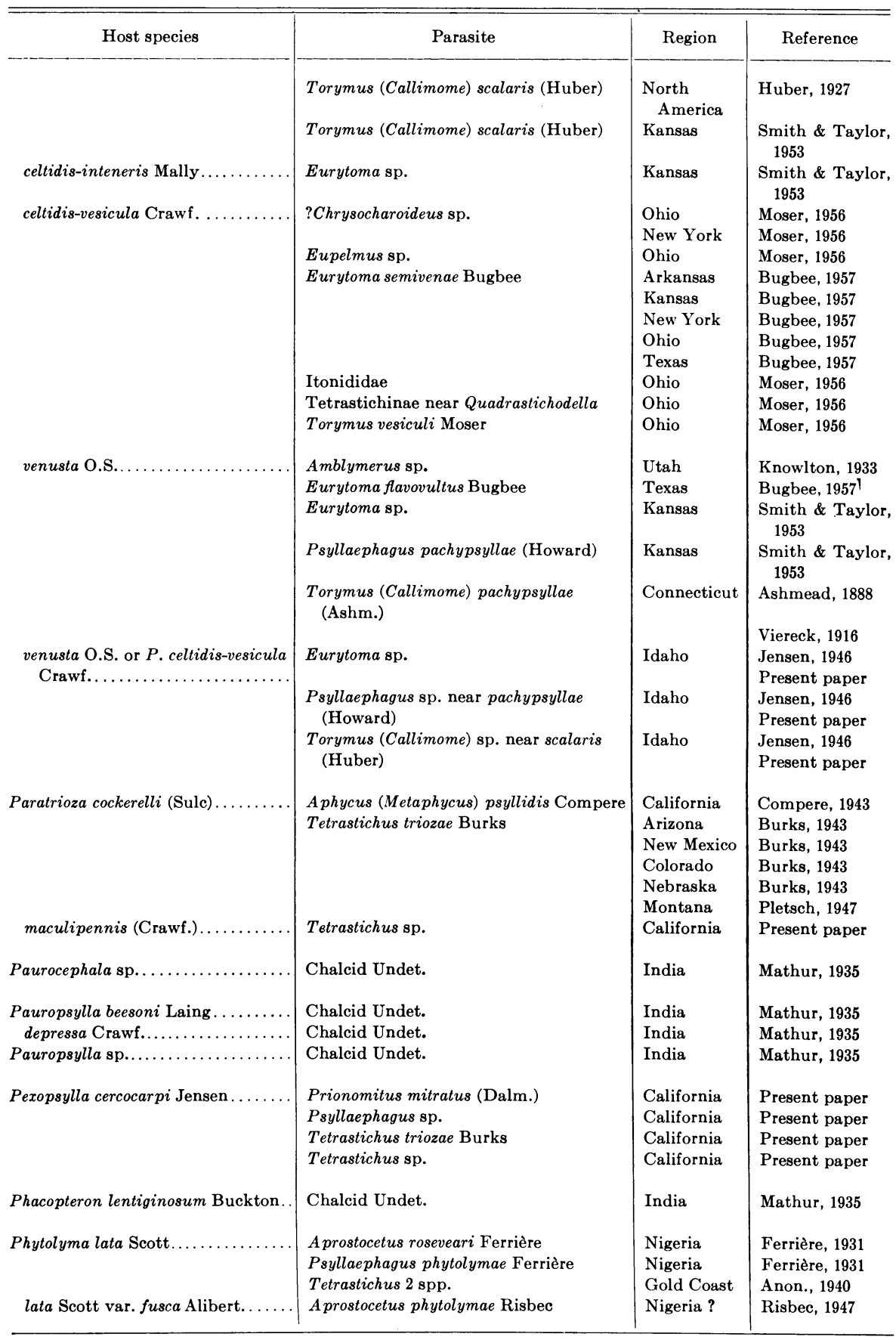


TABLE 2 (continued)

\begin{tabular}{|c|c|c|c|}
\hline Host species & Parasite & Region & Reference \\
\hline \multirow[t]{2}{*}{ Psylla americana Crawf............. } & Prionomitus mitratus (Dalm.) & California & Present paper \\
\hline & Prionomitus sp. & California & Jensen, 1951 \\
\hline \multirow[t]{2}{*}{ breviata Patch................... } & Prionomitus sp. & California & Jensen, 1951 \\
\hline & & & Present paper \\
\hline brevistigmata Crawf & Pteromalidae (Undet.) & California & Present paper \\
\hline crataegi (Schrank). & Prionomitus mitratus (Dalm.) & Switzerland & Wille, 1950 \\
\hline \multirow[t]{2}{*}{ floccosa Patch...................... } & Prionomitus sp. & California & Present paper \\
\hline & Pteromalidue (Undet.) & California & Present paper \\
\hline \multirow{2}{*}{ foersteri Flor. } & Endopsylla agilis de Meijere & Holland & de Meijere, 1907 \\
\hline & Endopsylla agilis de Meijere & England & $\begin{array}{l}\text { Bagnall and } \\
\text { Harrison, } 1924\end{array}$ \\
\hline mali (Schmidb.)., ............. & Endopsylla agilis? & Scotland & $\begin{array}{l}\text { Lal, } 1934 \\
\text { Barnes, } 1954\end{array}$ \\
\hline \multirow[t]{2}{*}{ melanoneura Först............. } & $\begin{array}{l}\text { Prionomitus sp. } \\
\text { Endopsylla agilis? }\end{array}$ & $\begin{array}{l}\text { Poland } \\
\text { Scotland }\end{array}$ & $\begin{array}{l}\text { Woroniecka, } 1928 \\
\text { Lal, } 1934 \\
\text { Barnes, } 1954\end{array}$ \\
\hline & Prionomitus mitratus (Dalm.) & Switzerland & Wille, 1950 \\
\hline \multirow{8}{*}{ 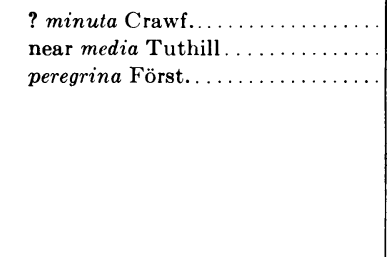 } & Psyllaephagus sp. & California & Present paper \\
\hline & Prionomitus mitratus (Dalm.) & California & Present paper \\
\hline & Endopsylla agilis? & Scotland & Lal, 1934 \\
\hline & Asaphes vulgaris Wa!ker & Scotland & $\begin{array}{l}\text { Barnes, } 1954 \\
\text { Lal, } 1934\end{array}$ \\
\hline & Lygocerus semiramosus Kieff. & Scotland & Lal, 1934 \\
\hline & Pachyneuron psyllaephaga Mani & Scotland & Lal, 1934 \\
\hline & Platygaster sp. & Scotland & Lal, 1934 \\
\hline & Prionomitus mitratus (Dalm.) & Scotland & Lal, 1934 \\
\hline \multirow{10}{*}{$\begin{array}{l}\text { pyri }(\mathrm{L} .) \ldots \ldots \ldots \ldots \ldots \ldots \ldots \\
\text { pyricola Först. } \ldots \ldots \ldots \ldots \ldots \ldots\end{array}$} & Prionomitus mitratus (Dalm.) & Switzerland & Wille, 1950 \\
\hline & Endopsylla agilis? & Scotland & Lal, 1934 \\
\hline & Encyrtus sp. & Russia & Barnes, 1954 \\
\hline & Metallon (Trechnites) psyllae Ruschka & Germany & $\begin{array}{l}\text { Yakhontov, } 1929 \\
\text { Ruschka, } 1923\end{array}$ \\
\hline & Pachyneuron sp. & Italy & $\begin{array}{l}\text { Kuscnka, } 1923 \\
\text { Golfari, } 1937\end{array}$ \\
\hline & & & Grandi, 1951 \\
\hline & Prionomitus mitratus (Dalm.) & Switzerland & Wille, 1950 \\
\hline & Psylledontus insidiosus Crawf. & New York & Crawford, 1911 \\
\hline & & Connecticut & $\begin{array}{l}\text { Anon., } 1931 \\
\text { Garman \& }\end{array}$ \\
\hline & & & Townsend, 1941 \\
\hline \multirow[t]{3}{*}{ pyrisuga Först.. } & Prionomitus mitratus (Dalm.) & France & Ferrière, 1926 \\
\hline & Prionomitus mitratus (Dalm.) & Switzerland & Brocher, 1926 \\
\hline & & & Wille, 1950 \\
\hline retamae Put. & Prionomitus mitratus $(\mathrm{D} \varepsilon$ & Spain & Marcet, 1926 \\
\hline ribesiae (Crawf & Prionomitus mitratus (Dalm.) & New Mexico & Jensen, 1956 \\
\hline tenuata Jensen & Prionomitus mitratus (Dalm.) & California & Jensen, 1951 \\
\hline Psylla sp... & Chalcid Undet. & India & Mathur, 1935 \\
\hline Psylla sp.. & Prionomitus sp. & Iran & Kiriukhin, 1946 \\
\hline Psylla sp.. & Thysanus (Signiphora) noacki (Ashm.) & Brazil & Ashmead, 1900 \\
\hline \multirow{2}{*}{$\begin{array}{l}\text { Psyllopsis fraxini (L.) } \ldots \ldots \ldots \ldots \ldots \\
\quad \text { fraxinicola Först................ }\end{array}$} & Bremia sp. (Itonididae) & Germany & Kieffer, 1901 \\
\hline & Cercobelus jugaeus Walker & Scotland & Lal, 1934 \\
\hline \multirow{3}{*}{$\begin{array}{l}\text { Rhinocola eucalypti Mask........... } \\
\quad \text { populi Laing................... }\end{array}$} & Pteroptrix maskelli Ashm. & New Zealand & Gourlay, 1930 \\
\hline & Psyllaephagus cellulatus Waterston & Mesopotamia & Waterston, 1922 \\
\hline & Pachyneuron crassiculme Waterston & Mesopotamia & Waterston, 1922 \\
\hline Syntomoza magna Kuwayama. & Psyllencyrtus syntomozae Tach. & Japan & Tachikawa, 1955 \\
\hline
\end{tabular}


TABLE 2 (continued)

\begin{tabular}{|c|c|c|c|}
\hline Host species & Parasite & Region & Reference \\
\hline Tenaphalara acutipennis Kuw... & Chalcid Undet. & India & Mathur, 1935 \\
\hline \multirow[t]{2}{*}{ Trioza alacris Flor............... } & Aphidius polygoni Marsh & Germany & Wagner, 1929 \\
\hline & Psyllaephagus femoralis Borelıi & Italy & Borelli, 1920 \\
\hline albifrons Crawf. & Tetrastichus triozae Burks & Cal'fornia & Present paper \\
\hline \multirow[t]{2}{*}{ 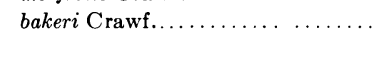 } & Psyllaephagus sp. & California & Present paper \\
\hline & Tetrastichus sp. & California & Present paper \\
\hline \multirow[t]{3}{*}{ beameri Tuthill. } & Prionomitus mitratus (Dalm.) & California & Present paper \\
\hline & Psyllaephagus pachypsyllae (Howard) & California & Present paper \\
\hline & Tetrastichus triozae Burks & California & Present paper \\
\hline \multirow[t]{3}{*}{ centranthi Vallot.............. } & Agonioneurus pictus Först. & France & André, 1878 \\
\hline & Encyrtus triozae André & France & André, 1878 \\
\hline & Tetrastichus obscuratus André & France & André, 1878 \\
\hline \multirow[t]{3}{*}{ citri Laing. } & Encyrtus pulvinatus Waterston & Kenya & Waterston, 1922 \\
\hline & Tetrastichus dryi Waterston & Kenya & Waterston, 1922 \\
\hline & Tetrastichus sicarius Silvestri & Kenya & Waterston, 1922 \\
\hline diospyri (Ashm.). & Psyllaephagus trioziphagus (Howard) & $\begin{array}{l}\text { Washington, } \\
\text { D.C. }\end{array}$ & Howard, 1885 \\
\hline fletcheri minor Crawf. & Chalcid Undet. & India & Mathur, 1935 \\
\hline gardneri Laing........ & Chalcid Undet. & India & Mathur, 1935 \\
\hline hirsuta (Crawf.). & Chalcid Undet. & India & Mathur, 1935 \\
\hline jambolanae Crawf. & Chalcid Undet. & India & Mathur, 1935 \\
\hline magnoliae (Ashm.). & Psyllaephagus solus (Howard) & Florida & Howard, 1885 \\
\hline malloticola (Crawf.). & Chalcid Undet. & India & Mathur, 1935 \\
\hline maura Först.. & Tetrastichus sp. & California & Present paper \\
\hline merwei Pettey. & Undetermined chalcid & South Africa & Merwe, 1923 \\
\hline minuta Crawf. & Tetrastichus triozae Burks & California & Present paper \\
\hline minuta arizonae Aulm. & Aphycus sp. & California & Present paper \\
\hline urticae (Linn.) & Tetrastichus obscuratus André & Italy & Grandi, 1953 \\
\hline viridula Zett... & Tetrastichus sp. & Latvia & Ozols, 1925 \\
\hline vitreoradiata (Mask.). & Encyrtidae Undet. & New Zealand & Carter, 1949 \\
\hline Trioza sp............. & Chalcid Undet. & India & Mathur, 1935 \\
\hline Trioza sp. . & Tetrastichus gelastus Burks & Florida & Burks, 1943 \\
\hline Trioza sp. & Tetrastichus dryus Burks & Montana & Burks, 1943 \\
\hline Trioza sp... & Tetrastichus triozae Burks & Washington & Burks, 1943 \\
\hline \multicolumn{4}{|l|}{ Psyllidae (undetermined) } \\
\hline Psyllid gall (? Trioza sp.). . & Cheiloneurus praenitens Waterston & Jamaica & Waterston, 1922 \\
\hline Psyllid on Cinnamomum sp.. & Psyllaephagus iwayaensis Ishii & Japan & Ishii, 1928 \\
\hline "Bred out of Eucalyptus". & Psyllaephagus metallicus (Girault) & Australia & Girault, $1915 a$ \\
\hline Gall-making psyllid ............ & Psylledontus secundus Girault & Ceylon & Girault, 1915 \\
\hline Psyllid on Elaeagnus umbellata.... & Psylledontus viridiscutellatus Ishii & Japan & Ishii, 1928 \\
\hline Psyllid........................... & Mirocerus psyllae Ashm. & Ceylon & $\begin{array}{l}\text { Ashmead, } 1904 \\
\text { Gahan \& Peck } \\
1946\end{array}$ \\
\hline
\end{tabular}




\section{NEW RECORDS OF PARASITES REARED FROM PSYLLID} NYMPHS, WITH NOTES ON BIOLOGY

\section{Aphalara near angustipennis Crawford}

Parasites: Psyllaephagus sp.

Host plant: Artemisia dracunculus L.

Locality: Five miles east of Olive, Orange County, California

On July 9, 1942, a collection was made of adults, nymphs, and eggs. Two nymphs were parasitized and from each an adult of Psyllaephagus was reared.

\section{Aphalara suaedae Crawford}

Parasites: Psyllaephagus sp.

Host plant: Suaeda sp.

Locality: Three miles northwest of Indio, Riverside County, California

On June 27, 1952, a plant of Suaeda, heavily infested with nymphs and adults, was collected by R. F. Smith. Many of the nymphs were parasitized and from them a number of adult Psyllaephagus sp. were reared.

\section{Aphalaroida pitbecolobia Crawford}

Parasites: Psyllaephagus sp.

Host plant: Acacia greggii Gray.

Locality: Banning, Riverside County, California

On May 11, 1943, nymphs infesting the host plant "catsclaw" were caged on one branch to rear them to the adult stage. On May 26, most of the nymphs had become adults. At this time eight nymphs were obviously parasitized and eight adults of Psyllaephagus sp. were reared. Each one emerged through a hole in the dorsum of the caudal portion of the abdomen.

\section{Arytaina amorphae (Mally)}

Parasites: Cheiloneurus sp.; Psyllaephagus sp.

Host plant: Amorpha fructicosa L. (in bloom)

Locality: Eight miles west of Corona, Orange County, California

May 6,1943, living adults and 8 parasitized nymphs (on the upper surface of the leaves) were collected. No living nymphs were found. On May 8, one adult parasite emerged through a hole in the abdomen of one nymph. On May 19, two more adult parasites emerged. All three were Cheiloneurus sp. Host plant: Amorpha fructicosa L. (beginning to bloom)

Locality: Cajon Pass, San Bernardino County, California

June 16, 1942, adult psyllids were abundant but only a few living nymphs were found. One nymph was parasitized. The adult parasite, Psyllaephagus sp., emerged and had died by June 25.

\section{Arytaina aculeata Crawford}

Parasites: Psyllaephagus sp.

Host plant: Cercocarpus betuloides Nutt.

Locality: San Timoteo Canyon, Riverside County, California

April 7, 1943, nymphs were found feeding in the axils of the leaves and on tender shoots and also at the base of the flower stems. A cloth sleeve cage was placed on the branch to prevent dispersal of the adults as they emerged. On May 15, practically all psyllids in the cage had reached the adult stage. 
A single parasitized nymph was placed in a glass vial. The adult parasite had emerged and was still alive May 19.

\section{Arytaina bicolor Jensen}

Parasites: Prionomitus sp.; Tetrastichus sp.

Host plant: Ceanothus cuneatus (Hook.) Nutt.

Locality: Mt. Diablo, Contra Costa County, California

May 25, 1949, adults were emerging in large numbers. Nymphs were common and occurred on the leaves which rolled upward and inward as a result of the nymphal feeding. The nymphs were concealed in the rolled leaves. The feeding of the nymphs also caused the infested leaves to become chlorotic and smaller than normal. The toxic effect of nymphal feeding was local and did not influence the growth of noninfested leaves of the same fascicle.

Several nymphs were found to be parasitized even though they were in the rolled leaves. Eight males and one female Prionomitus sp. emerged from the parasitized nymphs. One of these emerged June 3 and the others by June 5 . A single psyllid nymph was observed in the field to have a parasite larva attached to the outside venter of the body. The nymph and larva were placed in a glass vial for observation and rearing, May 25. On May 31 the parasite larva began to pupate between the nymph and the wall of the vial. June 4 the parasite had not yet emerged and the pupa was black. The adult parasite emerged June 5 or June 6 and proved to be Tetrastichus sp.

\section{Arytaina ceanothi Crawford}

Parasites: Prionomitus mitratus (Dalm.); Tetrastichus triozae Burks;

Psyllaephagus sp.; Pachyneuron sp.

Host plant: Ceanothus crassifolius Torr.

Locality: Oak Glen, Riverside County, California

On March 30,1943, nymphs and adults were found to be extremely abundant on the host plant. The nymphs were feeding primarily at the base of the flowers. Several nymphs were parasitized and were caged in glass vials. Ten parasite adults emerged between April 12 and April 14. Of the seven which have been identified, one was Prionomitus mitratus, one was Pachyneuron sp., and five were Psyllaephagus sp.

Host plant: Ceanothus tomentosus Parry (in bloom)

Locality: Wildwood Canyon, Yucaipa, San Bernardino County, California

Living adults and nymphs and two parasitized nymphs were collected April 11, 1943. On April 24, two adult parasites had emerged and were still alive. These were Tetrastichus triozae and Psyllaephagus sp.

Host plant: Ceanothus integerrimus H. \& A.

Locality: Sequoia National Park, California (Elev. 4,500 ft.)

Living nymphs were collected May 18, 1949. One nymph was parasitized, but was still active enough to walk a little on May 21. The parasite adult, Prionomitus sp., emerged June 4 through a circular hole in the dorsum of the abdomen.

Host plant: Ceanothus cuneatus (Hook.) Nutt.

Locality: Mt. Diablo, Contra Costa County, California

May 25, 1949, adults were common but no living nymphs were found. A single parasitized nymph was caged and the adult parasite, Psyllaephagus sp., emerged the same day. 


\section{Arytaina essigi Jensen}

Parasites: Prionomitus mitratus (Dalm.)

Host plant: Ceanothus tomentosus Parry

Locality: San Timoteo Canyon, Riverside County, California

On February 26, 1943, several nymphs and adults of this species were collected. The nymphs occurred at or just below the ground level feeding on the main woody branches of the bush. In addition, a few were found just above ground in a sheltered area under some loose bark. Several nymphs were caged singly in vials and provided with food. One nymph had remained active in the vial for two weeks and then became inactive. On April 21, 1943, one adult parasite of Prionomitus mitratus emerged through a hole in the dorsum of the abdomen. Three days later a second parasite emerged from the same nymph.

\section{Arytaina fuscipennis Crawford}

Parasites: Prionomitus mitratus (Dalm.)

Host plant: Ceanothus velutinus Dougl.

Locality: Mountains east of Bountiful, Davis County, Utah (Elev. 8,000 ft.)

July 1, 1943, adult psyllids were common on the foliage of the host plant and nymphs occurred on the main branches just below the surface of the soil or at places where the branches rested against the soil. Most of the nymphs were in their last instar and many of them were parasitized. These were caged in vials to await parasite emergence. By July 22, 1943, thirty adult Prionomitus mitratus had emerged and were dead.

\section{Arytaina insolita Tuthill}

Parasites: Prionomitus mitratus (Dalm.)

Host plant: Ceanothus velutinus Dougl.

Locality: Mountains east of Bountiful, Davis County, Utah (Elev. 8,000 ft.)

Living adults and nymphs were collected July 1, 1943. A single parasitized nymph was held in a glass vial. The adult parasite emerged and had died by July 22.

\section{Arytaina minuta Crawford}

Parasites: Prionomitus mitratus (Dalm.) ; Tetrastichus triozae Burks Host plant: Ceanothus crassifolius Torr.

Locality: North ridge of Moreno Valley, Riverside County, California

April 7, 1943, several nymphs were collected and caged in vials for rearing to the adult stage. On April 11, several of the caged nymphs were beginning to develop the swollen appearance characteristic of parasitized nymphs. On April 25, four adult parasites emerged through the dorsum of the abdomen of four nymphs. On April 30, two more parasites emerged. All proved to be Prionomitus mitratus.

Host plant: Ceanothus crassifolius Torr.

Locality: Beaumont, Riverside County, California

April 13, 1943, parasitized nymphs were placed in rearing vials. Between May 1 and May 3, eight Prionomitus mitratus adults emerged from the parasitized nymphs through holes in the dorsum of the abdomen and one Tetrastichus triozae emerged through a smaller hole beneath the head.

\section{Arytaina "robusta" Crawford}

Parasites: Prionomitus mitratus (Dalm.); Prionomitus sp.; Psyllaephagus 


\section{Host plant: Ceanothus tomentosus Parry}

Locality: Yucaipa, San Bernardino County, California

Adults were abundant and a few living nymphs occurred on the leaves May 14, 1943. Several nymphs were placed in rearing vials. On May 16, one nymph, which had been active on May 14, had become immobile, was somewhat swollen and had turned a light brown color. The movement of a parasite larva could be discerned inside the nymphal body. The date of the adult parasite's emergence was not determined. It was identified as Prionomitus mitratus.

Host plant: Ceanothus leucodermis Green

Locality: Sequoia National Park, Tulare County, California (Elev. 6,400 ft.)

Adults and nymphs were collected May 18, 1949, from the new growth of a single bush growing against some large boulders with a south exposure. The other bushes in the vicinity had made no new growth as yet because of the near freezing temperatures at night and the prevalence of snow over the area. One nymph was parasitized and the adult parasite emerged June 3 through a circular hole $0.5 \mathrm{~mm}$. in diameter in the dorsum of the abdomen. The parasite was Psyllaephagus sp.

Host plant: Ceanothus velutinus Dougl.

Locality: Five miles south of Sierraville, Sierra County, California

Adults and nymphs were collected June 26, 1949, and several nymphs were reared to the adult stage. The nymphs occurred primarily in the angle formed by two leaves which were in contact with each other-in most cases due to the activities of a leaftying caterpillar.

On July 5, four nymphs were observed to be swollen as if parasitized. They had been normal in appearance when collected June 26. Only two adult parasites were recovered. On July 15 one had emerged and was dead. A second parasite became an adult inside the psyllid nymph, but died without emerging. Both were males of Prionomitus sp.

Host plant: Ceanothus cordulatus Kell.

Locality: Buck's Lake, Plumas County, California (Elev. 5,500 ft.)

Adults and nymphs were collected June 27, 1949. A single parasitized nymph was placed in a rearing vial. Emergence of the adult parasite from the psyllid nymph was observed July 12, 1949. A round hole was chewed in the venter of the abdomen of the psyllid. Several attempts were made by the parasite to emerge before the hole was made large enough to allow passage of the head and antennae. The material bitten off in making the emergence hole was swallowed by the parasite, Prionomitus sp.

\section{Calopbya californica Schwarz}

Parasites: Tetrastichus triozae Burks

Host plant: Rhus ovata Wats.

Locality: San Timoteo Canyon, Riverside County, California

On March 26, 1942, branches of sumac, with the flower buds still unopened, were taken into the laboratory at San Bernardino. The nymphs were found feeding only on the floral buds, and always had their heads directed toward the bud attachment to the peduncle.

Several of the nymphs were parasitized when collected March 26. Two adults of Tetrastichus triozae emerged April 2, while a third had broken a 
hole in the middle of the psyllid nymph's dorsum and could be seen moving its legs. This parasite emerged between 8:00 a.m. and 9:00 a.m. on April 3, and, although its wings were not yet dry at 9:00 a.m. it was copulating with one of the adults which had emerged the day before.

\section{Calophya nigrella Jensen}

Parasites: Tetrastichus triozae Burks

Host plant: Rhus trilobata Nutt.

Locality: San Timoteo Canyon, Riverside County, California

On February 26, 1943, most of the Rhus trilobata bushes were still dormant but a few were beginning to blossom and produce very young leaves. Several adults and nymphs were collected, the nymphs being scattered sparsely over the woody branches. None occurred on the flowers or leaves.

Among the nymphs caged on twigs were several which appeared to be parasitized. Only one parasite adult was obtained and it did not emerge until March 21, 1943. It was Tetrastichus triozae.

\section{Calophya triozomima Schwarz}

Parasites: Tetrastichus triozae Burks

Host plant: Rhus trilobata Nutt.

Locality: Mill Creek Canyon, San Bernardino County, California

On April 16, 1943, nymphs and adults occurred on the young leaves and to a lesser extent on the blossoms. Eight parasitized nymphs were caged and the emerging parasites were all Tetrastichus triozae. They emerged between April 16 and April 28. Two of the parasites did not emerge through holes cut for that purpose in the dorsal surface of the nymph, but had eaten away the ventral surface of the nymph's body and merely pushed the shell up enough to crawl out.

\section{Euphalerus vermiculosus Crawford}

Parasites: Undet.

Host plant: Ceanothus cordulatus Kell.

Locality: Lake Almanor, Plumas County, California

Adults and nymphs were collected July 13, 1949. On July 25, two of the nymphs held in a rearing cage were observed to be swollen as if parasitized. The adult parasites emerged, but have not yet been determined.

\section{Eupbalerus vermiculosus beameri Tuthill}

Parasites: Tetrastichus triozae Burks

Host plant: Ceanothus leucodermis Green

Locality: Big Pines Camp, Los Angeles County, California

Branches, infested with nymphs, were cut June 21, 1942, and taken to San Bernardino for rearing and observation. The nymphs feed on the under side of the leaves where they form a cottony, waxy cell which completely surrounds them and, when fully formed, is approximately $3.5 \mathrm{~mm}$. in outside diameter. This flocculent mass is produced by the nymph as wax threads, coming from the circum-anal area and the basal portion of the caudal segments. Freshly produced wax threads are loosely curled among themselves, shiny grayish white in color and easily discernible as individual threads. The flocculence of old and abandoned cells is more compact, whiter, and the component threads are not so apparent. 
As the cottony mass becomes larger, both above and below the nymphs, the cell, formed by the body movements, is gradually forced away from the surface of the leaf-particularly the caudal portion. The nymph is finally situated at about a 30-degree angle to the leaf surface. The wax mass does not adhere to the leaf except over a small area around the feeding site. The early nymphal exuviae are gradually carried away with the newly formed wax threads and appear in the surface portion of the mass.

It is not known whether nymphs become parasitized while still in the wax cell or whether this occurs after they leave the cell or just before they become adults. Two adults of Tetrastichus triozae emerged July 1, 1942, from nymphs held in glass vials.

\section{Eupbyllura arbuti Schwarz}

Parasites: Psyllaephagus arbuticola Gahan and Waterston; Psyllaephagus

sp.

Host plant: Arbutus menziesii Pursh.

Locality: El Cerrito, Contra Costa County, California

A branch of the host tree, infested with nymphs, was brought to the writer by Mrs. P. L. Small in May, 1949. One nymph was parasitized by Psyllaephagus sp. Gahan and Waterston (1926) described as new the species Psyllaephagus arbuticola from specimens reared from a psyllid on Arbutus in San Mateo and Alameda Counties, California, and specimens reared from Euphyllura arbuti at Stanford University, California. It is probable that the specimen reported in the present work will prove to be Psyllaephagus arbuticola.

\section{Eupbyllura arctostapbyli Schwarz}

Host plant: Arctostaphylos sp.

Locality: Lake Almanor, Plumas County, California

On July 13, 1949, nymphs occurred under the conspicuous, white, waxy masses which they produce. These occurred almost exclusively where two leaves were held against each other-usually due to the activities of leaf tiers. Nymphs were also feeding on the woody main stems, but only at places where the bark was cracked and the nymphs could get beneath part of the bark. On July 25 three parasitized nymphs were noted in the rearing tubes. The emerging adult parasites are as yet undetermined.

Pacbypsylla venusta O. S. and P. celtidis-vesicula Crawford

Parasites: Torymus (Callimome) sp. near scalaris Huber; Psyllaephagus sp. near pachypsyllae (Howard); Eurytoma sp.

Host plant: Celtis douglasi Planchon

Locality: Lucile, Idaho County, Idaho

On October 16, 1941, adults of Pachypsylla venusta were common on the host plant which also carried many of the galls produced on the leaf petioles and the base of the leaves. On the same plant were many leaf galls infested with nymphs of Pachypsylla celtidis-vesicula. The adults of this species had not begun to emerge.

Foliage, bearing galls of both species, was taken to San Bernardino, California, where the galls were kept dry and at room temperature. Adults of both species were reared, venusta emerging as late as February, 1942, and celtidis-vesicula as late as the end of December, 1941. 
Examination of the nymph-infested galls of both venusta and celtidisvesicula revealed that several galls each contained one hymenopterous larva which apparently developed outside of the psyllid nymph but fed on the nymph. Nothing was left of the psyllid nymph in most galls except the more heavily sclerotized portions of the exoskeleton. The skin of one nymph was entire, but was collapsed as if the body contents had been sucked out.

Six adult parasites emerged between February and April 20, 1942, and an additional eight parasites emerged after April 20. Both psyllid species had been parasitized, but the material became mixed and it could not be determined from which host species the respective parasites had emerged.

\section{Paratrioza maculipennis (Crawford)}

Parasites: Tetrastichus sp.

Host plant: Convolvulus sp.

Locality: Palo Alto, Santa Clara County, California

Nymphs and adults were collected by E. S. Sylvester and D. D. Jensen on October 15,1948 . The nymphs were very abundant on the upper portions of the roots, 1 to 2 inches below the surface of the soil, on the main stem of the plant and 1 to 2 inches up the foliage branches above the ground. The exit holes of parasites were noted in a number of nymphs and 15 adults of Tetrastichus sp. were reared from nymphs taken into the laboratory at Berkeley. Emergence of the parasites occurred between October 15 and October 26, 1948.

\section{Pexopsylla cercocarpi Jensen}

Parasites: Tetrastichus triozae Burks, Tetrastichus sp., Prionomitus mitratus

(Dalm.), Psyllaephagus sp.

Host plant: Cercocarpus betuloides Nutt.

Locality: San Timoteo Canyon, Riverside County, California

Adults and nymphs, including two parasitized nymphs, were collected February 2, 1943. The adult parasite emerged from one nymph February 6 and from the second nymph February 10 . They were identified as Tetrastichus sp. Both emerged through circular holes in the dorsum at the juncture of head and thorax.

Host plant: Cercocarpus ledifolius Nutt.

Locality: Wrightwood, San Bernardino County, California

Adults and nymphs were collected on April 28, 1943. On May 2, three nymphs were suspected of being parasitized. Two of them were obviously swollen and the exoskeletons were stiff. The third nymph was still of normal light green color, and, though slightly swollen, the exoskeleton was pliant. Parasites emerged from all three nymphs by May 17, and were identified as Tetrastichus triozae, Prionomitus mitratus, and Psyllaephagus sp.

\section{Psylla americana Crawford}

Parasites: Prionomitus mitratus (Dalm.); Prionomitus sp.

Host plant: Salix sp.

Locality: Mill Creek Canyon, San Bernardino County, California

Adults and nymphs were collected in large numbers April 1, 1943. Two parasitized nymphs were caged. The adult parasites emerged from one nymph April 8 and from the second nymph April 10. They were identified as Prionomitus sp. (Jensen, 1951). 
Host plant: Salix sp.

Locality: Bar Bear, San Bernardino County, California (Elev. 6,000 ft.) On June 7, 1942, adults and nymphs were abundant on the host plant. Leaves, infested with nymphs, were taken to the laboratory for rearing. Three weeks later several nymphs were observed to have been parasitized, but the parasites except one had emerged and escaped. In this case, the adult parasite had died while still in the body of the psyllid nymph. The parasite had succeeded in cutting an adequately large emergence hole in the dorsal surface of the abdomen, but died without emerging. It was identified as Prionomitus mitratus.

Host plant: Salix sp.

Locality: Hobart Mills, Nevada County, California

Adults and nymphs were present in small numbers June 26, 1949. An adult of Prionomitus sp. emerged from one nymph.

\section{Psylla breviata Patch}

Parasites: Prionomitus sp.

Host plant: Salix sp.

Locality: Hobart Mills, Nevada County, California

Several adults and nymphs, including some parasitized nymphs, were collected June 26, 1949 (Jensen, 1951). One adult of Prionomitus sp. emerged from one psyllid nymph, and the parasite in a second nymph became an adult but died within the psyllid nymph.

\section{Psylla brevistigmata Patch}

Parasites: Undetermined Pteromalidae

Host plant: Cercocarpus betuloides Nutt.

Locality: Kaweah, Tulare County, California

Adults and nymphs were collected May 21, 1952, the nymphs occurring in the protected places provided by the severely rolled leaves. The malformation apparently resulted from the feeding of the nymphs.

On May 27, an adult parasite (undetermined Pteromalidae) emerged through a hole cut in the dorsal surface of a nymph's body.

\section{Psylla floccosa Patch}

Parasites: Prionomitus sp.; Undetermined Pteromalidae

Host plant: Alnus sp.

Locality: Buck's Lake, Plumas County, California

On July 14, 1949, nymphs were numerous on the terminal shoots and on the young sucker growth of the host plant. A few of the nymphs were parasitized and these were placed in rearing vials. On July 25 five parasites had emerged, two being alive, and the other three dead. One female and two males were Prionomitus sp.; one male and one female are listed as undetermined Pteromalidae.

\section{Psylla near media Tuthill}

Parasites: Prionomitus mitratus (Dalm.)

Host plant: Cercocarpus betuloides Nutt.

Locality: Forest Home, San Bernardino County, California

March 6, 1943, twenty last-instar nymphs were collected and placed in glass vials with leaves of the host plant. Twelve adult psyllids were reared from these nymphs. Three nymphs proved to be parasitized and adult 
parasites were reared from two of these. One parasite emerged through a hole cut in the dorsum of the nymph's abdomen March 24 and the second parasite emerged March 27. Both were Prionomitus mitratus.

\section{Psylla minuta Crawford}

Parasites: Psyllaephagus sp.

Host plant: Purshia tridentata DC.

Locality: Wrightwood, San Bernardino County, California

Adults and nymphs occurred on the host plant in large numbers April 28, 1943, in the presence of small numbers of Psylla coryli. Several nymphs were parasitized and are presumed to be nymphs of minuta. By May 17, five adult parasites of Psyllaephagus sp. had emerged from their host nymphs and were dead. Each had emerged through a hole cut in the dorsum of the nymph's abdomen.

\section{Psylla ribesiae (Crawford)}

Parasites: Prionomitus mitratus (Dalm.)

Host plant: Ribes sp. (Wild currant)

Locality: Espanola, Rio Arriba County, New Mexico

Among the nymphs and adults collected on June 3, 1943, were two parasitized nymphs which were placed in rearing vials. On June 20, 1943, one parasite had emerged and was lost. The parasite in the second nymph had become an adult and was moving within the shell of the psyllid nymph. However, this parasite died without emerging. It was removed and identified as Prionomitus mitratus (Jensen, 1956).

\section{Psylla tenuata Jensen}

Parasites: Prionomitus sp.

Host plant: Salix laevigata Bebb.

Locality: Colton, San Bernardino County, California

On March 13, 1943, nymphs were feeding on the staminate catkins with their heads directed toward the catkin stem. What appeared to be a parasitic wasp was observed to drag a psyllid nymph on its back by grasping the back of the nymph with its hind legs. It was not determined whether or not the parasite oviposited in the nymph, but the nymph appeared to be paralyzed for several minutes and then began to crawl. The psyllid and the parasite were caged for subsequent observation, but were lost before additional information was obtained.

On April 7, 1943, eight parasitized nymphs were collected from the same host tree examined March 13. Between April 18 and April 24 seven adult parasites emerged. The emergence hole was cut in the dorsum of the abdomen in six of the nymphs and in the venter of the seventh. They were identified as an undescribed species of Prionomitus (Jensen, 1951).

\section{Trioza albifrons Crawford}

Parasites: Tetrastichus triozae Burks

Host plant: Urtica sp.

Locality: Live Oak Canyon, San Bernardino County, California

Adults, nymphs, and eggs were common on the terminal growth of the host plant February 2, 1943. One nymph was parasitized and attached to the under side of the leaf by a network of fine, dry threads. The entire venter 
of the nymph had been eaten away so that the parasite was in direct contact with the leaf. The parasite adult, Tetrastichus triozae, emerged February 10 by pushing the nymphal shell away from the leaf a little instead of through a hole in the dorsum as is usually done.

\section{Trioza bakeri Crawford}

Parasites: Tetrastichus sp.; Psyllaephagus sp.

Host plant: Rhamnus crocea var. ilicifolia Green

Locality: Kaweah, Tulare County, California

Living adults and nymphs were collected May 21, 1952. The feeding of the nymphs apparently caused the leaves to roll, thus providing a somewhat protected place for the nymphs. Several parasitized nymphs were placed in glass vials, and by May 27 eight specimens of Tetrastichus sp. and three specimens of Psyllaephagus sp. had emerged from the nymphs.

\section{Trioza beameri Tuthill}

Parasites: Prionomitus mitratus (Dalm.); Tetrastichus triozae Burks; Psyllaephagus pachypsyllae (Howard)

Host plant: Rhamnus californica Esch.

Locality: Mill Creek Canyon, San Bernardino County, California

A few last-instar nymphs occurred on the under side of the leaves April 16, 1943. Two nymphs were parasitized and an adult Prionomitus mitratus emerged from one of them.

Host plant: Rhamnus californica Esch.

Locality: Wrightwood, San Bernardino County, California (Elev. 6,000 ft.)

On June 21, 1942, adults, nymphs, and eggs occurred on the host plant. Several nymphs were parasitized and between June 25 and June 28, an adult Tetrastichus triozae and one adult Psyllaephagus pachypsyllae emerged.

\section{Trioza maura Foerster}

Parasites: Tetrastichus sp.

Host plant: Salix sp.

Locality: Sequoia National Park, Tulare County, California (Elev. 6,500 ft.)

On September 14, 1948, nymphs were present in large numbers and usually occurred on the under surface of the leaves. The nymphs apparently had a toxic effect upon the plant, because a yellow blotch developed in the leaf around the feeding site of each nymph.

Some parasitized nymphs were collected in glass vials and held for emergence of the adult wasps. By September 22 two adult parasites had emerged and a third was observed during its emergence. It was lying on its back and had cut a round hole in the dorsum of the nymph's thorax. Actual emergence from the nymph, after the hole was made, occupied only one minute. Five parasites were reared and all proved to be Tetrastichus sp.

\section{Trioza minuta arizonae Aulmann}

Parasites: Aphycus sp.

Host plant: Salix lasiandra Benth.

Locality: Five miles east of Olive, Orange County, California

Adults and nymphs were collected from the leaves July 9, 1942. Adult psyllids were not reared from any of the nymphs, so the evidence is only presumptive that the nymphs were $T$. minuta arizonae.

Three adults of Aphycus sp. were reared from a single parasitized nymph. 


\section{Trioza minuta Crawford?}

\section{Parasites: Tetrastichus triozae Burks}

Host plant: Salix sp.

Locality: Colton, San Bernardino County, California

On April 16,1943, three triozine nymphs were collected on a single leaf of the host plant. The leaf was placed in a vial to rear the adult psyllids. A female adult of Trioza minuta developed from one nymph, but the other two nymphs proved to be parasitized by Tetrastichus triozae. Emergence of the adult parasites occurred through the dorsum of the thorax in each case.

\section{ACKNOWLEDGMENTS}

Most of the parasites reared during this investigation were identified by A. B. Gahan and B. D. Burks of the Division of Insect Detection and Identification, U. S. National Museum. The balance were identified by F. E. Skinner, University of California, Berkeley. Grateful acknowledgment is made for this service and for the corrections made by Mr. Skinner in the manuscript.

\section{LITERATURE CITED}

Alibert, $\mathrm{H}$.

1947. Phytolyma lata Scott var. fusca var. n., Psyllidae, vivant sur iroko (Chlorophora excelsa). Agron. Trop. 2:165-66.

ANDRÉ, EDMOND

1878. Memoire pour servir a l'histoire de la Trioza centranthi Vallot. Ann. Soc. Ent. France 8:77-86.

Anonymous

1931. Division of Entomology. 49th Ann. Rpt., New York Agr. Expt. Sta. 1929$30: 61-71$.

1940. Work of the entomologist. Gold Coast Dept. Agr., Rpt. 1939-40:3.

Ashmead, W. H.

1888. Descriptions of some unknown parasitic Hymenoptera in the collection of the Kansas State Agricultural College, received from Prof. E. A. Popenoe. Kansas Agr. Expt. Sta. Bul. 3, Appendix, pp. I-VIII.

1900. On the genera of the Chalcid-flies belonging to the subfamily Encyrtinae. U. S. Natl. Mus. Proc. 22:323-412.

1904. Classification of the superfamily Chalcidoidea. Carnegie Mus. Mem. 1:225-532.

BAGNALL, R.S., and H. HARRISON

1924. New British Cecidomyidae. V. Ent. Rec. and Jour. Variation. 36:38.

BARNES, H. F.

1930. Gall midges (Cecidomyidae) as enemies of the Tingidae, Psyllidae, Aleurodidae and Coccidae. Bul. Ent. Res. 21:319-29.

1954. Gall-midge larvae as endoparasites, including the description of a species parasitising aphids in Trinidad, B.W.I. Bul. Ent. Res. 45:769-75.

Borelli, NATALia

1920. Contributo alla conoscenze della vita nelle Galle dell' Alloro. Bol. Soc. Ent. Ital. 51:3-37.

BROCHER, F.

1926. Observations biologiques sur Psylla pyrisuga (Hemipt.). Ann. Soc. Ent. France 95:183-88.

Bugbee, R. E.

1957. Four new species of the genus Eurytoma from galls on hackberry (Chalcidoidea, Hymenoptera). Kansas Ent. Soc. Jour. 30:45-50.

BURKs, B. D.

1943. The North American parasitic wasps of the genus Tetrastichus. A contribution to the biological control of insect pests. U. S. Natl. Mus., Proc. 93:505-608. 
CALDWELL, J. S.

1938. The jumping plant lice of Ohio (Homoptera: Chermidae). Ohio. Biol. Survey Bul. 34 (vol. 6, no. 5) :228-81.

Carter, Myra W.

1949. The Pittosporum Chermid, Powellia vitreoradiata Mask. New Zeal. Jour. Sci. and Tech., sec. B, 31 (2):31-42.

Clausen, C.P.

1940. Entomophagous insects. 688 pp. MeGraw-Hill Book Co., Inc., New York, N.Y.

COMPERE, HAROLD

1943. A new species of Metaphycus parasitic on psyllids. Pan-Pacific Ent. 19:71-73.

CRAW FORD, J. C.

1911. Three new genera and species of parasitic Hymenoptera. U. S. Natl. Mus., Proc. 38: $87-90$.

FerRière, Ch.

1926. Un parasite de Psyllia pyrisuga. Ann. Soc. Ent. France 95:189-94.

1931. Notes on African Chalcidoidea. Bul. Ent. Res. 22:127-35.

Ferris, G. F., and Persis HyatT

1923. The life history of Euphyllura arbuti Schwarz (Hemiptera:Chermidae). Canad. Ent. 55:88-92.

GaHAN, A. B.

1925. A second lot of parasitic Hymenoptera from the Philippines. Philippine Jour. Sci. 27:83-109.

Gahan, A. B., and O. Peck

1946. Notes on some Ashmedian genotypes in the hymenopterous superfamily Chalcidoidea. Wash. Acad. Sci. Jour. 36:314-20.

GahAN, A. B., and J. Waterston

1926. Notes on Encyrtidae (Hym.-Chalcidoidea) bred from psyllids, with descriptions of a new species. Bul. Ent. Res. 15:373-75.

Garman, P. and J. F. Townsend

1941. Control of the Pear Psylla in Connecticut. Conn. Agr. Expt. Sta. Cir. 143:1-12.

GiraulT, A. A.

1915. New chalcidoid Hymenoptera. Ent. Soc. Amer. Ann. 8:279-284.

1915a. Australian Hymenoptera Chalcidoidea-VII. Mem. Queens Mus. 4:1-184.

GOLFARI, LAMBERTO

1937. Contributi alla conoscenza dell' Entomofauna del Pero (Pirus communis L.) Bol. Istit. Ent. Univ. Bologna 9:206-49.

Gourlay, E. S.

1930. Some parasitic Hymenoptera of economic importance in New Zealand. New Zeal. Jour. Sci. and Tech. $11: 339-43$.

GRANDI, Guido

1951. Introduzione allo studio dell' Entomologia. Vol. I. Edizioni Agricola-Bologna: 814-822.

1953. Aggiunte e correzioni, introduzione allo studio dell' Entomologia. Bol. Istit. Ent. Univ. Bologna 19:263-306.

HowARD, L. O.

1885. Descriptions of North American Chalcididae. U. S. Dept. Agr. Bur. Ent. Bul. $5: 15$.

HUBER, L. L.

1927. A taxonomic and ecological review of the North American chalcid flies of the genus Callimome. U. S. Nat. Mus., Proc. 70:1-114.

Husain, M. A., and L. D. NATH

1924. The life history of Tetrastichus radiatus parasitic on Euphalerus citri Kuw., and its hyperparasite. 5th Ent. Meeting, Pusa (Calcutta), Rpt. Proc. pp. 122-28.

1927. The citrus psylla (Diaphorina citri, Kuw.) Psyllidae: Homoptera. India Dept. Agr. Mem. 10:5-27.

IsHII, T.

1928. The Encyrtinae of Japan. Bul. Imp. Agr. Expt. Sta. Japan 3(2) :79-160.

JENSEN, D. D.

1946. A new Eupha?erus and notes on other species of Psyllidae from Idaho. Ent. Soc. Amer. Ann. 39:242-45. 
1951. The North American species of Psylla from willow, with descriptions of new species and notes on biology. Hilgardia $20(16): 299-324$.

1956. New species of Psylla from western United States and biological notes. Canad. Ent. 88:101-9.

KIEFFER, J. J.

1901. Cecidomyides d'Europe et d'Algerie. Ann. Soc. Ent. France 69:181-472.

KIRIUKHIN, G.

1946. Les insectes nuisibles au Pistacier en Iran. Ent. et Phytopath. Appl. (Teheran) $1: 8-24$.

KNOWLTON, G. F.

1933. Notes on injurious Utah insects. Utah Acad. Sci. Proc. 10:153, 159-62.

LAL, K. B.

1934. Insect parasites of Psyllidae. Parasitology 26:325-34.

LiENK, S. E.

1951. Revision of the genus Torymus in America north of Mexico. Ph.D. thesis (unpublished). Univ. Ill. 270 pp. 7 pl. (see Moser, 1956).

MANI, M. S.

1939. Chalcidoid and other parasites. Indian Jour. Ent. 1:84-85.

MASI, L.

1911. Contribuziono alla conoscenza dei calcididi Italiani. Bol. Lab. Zool. Gen. Agr., Portici 5:140-71.

MAThUR, R. N.

1935. On the biology of the Psyllidae (Homopt.). Indian Forest Records (New series) $1(2): 35-71$.

MeIJeRE, J. C. H. DE

1907. Eerste supplement op de nieuwe naamlijst van Nederlandsche Diptera. Tijdschr. Ent. 50:151-95.

MERCET, R. G.

1926. Adiciones a la fauna española de Encirtidos-6 ${ }^{a}$ nota (Hym. Chale.). Eos 2(4) :309-20.

MERWE, C. P. VAN DER

1923. The citrus Psylla (Trioza merwei Pettey). Union So. Africa Dept. Agr., Jour. $7(2): 135-141$.

Moser, J. C.

1956. A new species of Torymus (Hymenoptera:Torymidae) parasitic on Pachypsylla celtidis-vesicula Riley (Chermidae) with notes on its biology and other parasitoids attacking the same host at Columbus, Ohio. Kansas Ent. Soc. Jour. 29:57-62.

Muesebeck, C. F. W., K. V. Krombein, and H. K. Townes

1951. Hymenoptera of America north of Mexico. Synoptic catalog, U. S. Dept. Agr. Agr. Monog. 2:1-1420.

OzoLs, E.

1925. Zala burkanu lapu uts (Trioza viridula Zett.). Lauksaimniecibas parvaldes izdevums, Krajumm pie "Letas," Riga. 32 pp. Abstracted in: Rev. Appl. Ent., ser. A, 14:137.

Pletsch, D. J.

1947. The potato Psyllid, Paratrioza cockerelli (Sule), its biology and control. Montana Agr. Expt. Sta. Tech. Bul. 446:1-95.

RISBEC, J.

1947. Note au suject des parasites du Phytolyma lata. Agron. Trop. 2:167-69.

RÜBSAAMEN, $\mathrm{H}$.

1901. Bericht über meine Reisen durch die Tucheler Heide in den Jahren 1896 und 1897. Schrift. Naturf. Gesell. Danzig 10:79-148.

RUSChKa, Franz

1923. Beiträge zur Kenntnis einiger Encyrtidengattungen (Hym. Chalcid.). Verh. Zool. Bot. Ges. Wien 72:13.

Silvestri, F.

1915. Contributo alla conoscenza degli insetti dell' olivo dell' Eritrea e dell' Africa meridionale. Bol. Lab. Zool. Gen. Agr., Portici 9:240-334. 
SMITH, R. C., and R. S. TAYLOR

1953. The biology and control of the hackberry psyllids in Kansas. Kansas Ent. Soc. Jour. 26:103-15.

SPEYER, W.

1929. Der apfelblattsauger Psylla mali Schmidberger. Monogr. z. Pflanzenschutz No. 1. $127 \mathrm{pp}$.

1941. Endopsylla ?agilis de Meijere (Cecidomyidae) als Endoparasit von Psylla mali Schmidb. Arb. physiol. angew. Ent. 8:39-41.

TACHIKAWA, T.

1955. A new genus of Encyrtidae parasitic on a psyllid (Hymenoptera). Kontyu 23:63-67.

1956. A supplementary note on the genera of Encyrtidae parasitic on psyllids (Hymenoptera). Kontyu 24:173.

THOMPSON, W. R.

1944. A catalogue of the parasites and predators of insect pests. Imperial Inst. Ent. Parasite Service. Sec. 1, Parasite host eat. Part 3, Parasites of the Hemiptera: 1-149.

VIERECK, H. L.

1916. Guide to the insects of Connecticut, Part 3, Hymenoptera. State Zool. and Nat. Hist. Survey of Conn., Bul. 22:516.

VOSSELER, J.

1906. Eine Psyllide als Erzeugerin von gallen am Meulebaum. Zeitschr. Wiss. Insekten Biologie 2:276-285, 305, 316.

WAGNER, A. C. W.

1929. Schlupfwespen und ihre Wirte. Zuchtergebnisse von Hamburg Entomologen. Verh. Ver. Naturw. Unterhaltung Hamburg 20:17.

WALTON, C. J.

1944. The biology of Pachypsylla celtidis-gemma Riley and the relationship of the parasite to the bud of Celtis occidentalis. Fordham Univ. Doctor's Dissertation. 100 pp.

WATERSTON, J.

1922. On the Chalcid parasites of Psyllidae. Bul. Ent. Res. 13:41-58.

1923. Notes on parasitic Hymenoptera. Bul. Ent. Res. 14:103-108.

WILLE, H. P.

1950. Untersuchungen über Psylla piri L. und andere Birnblattsaugerarten im Wallis. Promotionsarbeit, Eidg. Tech. Hochsch. Zurich. 113 pp.

WORONIECKA, J.

1928. [Observations on the pests of cultivated plants that appeared in the district of Lublin and in part of the district of Kielce during the years 1926 and 1927.] Mem. Inst. Nat. Polon. Econ. Rur. Pulawy 9:216-251. Abstracted in: Rev. Appl. Ent., ser. A, 17:130-31.

YAKHONTOV, V. V.

1929. [List of pests of economic plants in the region of Bokhara and of their parasites and insect predators.] Trudui Shirabud. Opuitn. S.-Kh. Sta. Otdel Zasheh. Rast. No. 2, 46 pp. (See Thompson, 1944.)

ZANGHERI, SERGIO

1954. Nota sulla “Trioza urticae” L. (Homoptera, Psyllidae). Bol. Istit. Ent. Univ. Bologna 20:257-73. 

The journal Hilgardia is published at irregular intervals, in volumes of about 600 pages. The number of issues per volume varies.

Subscriptions are not sold. The periodical is sent as published only to libraries, or to institutions in foreign countries having pub. lications to offer in exchange.

You may obtain a single copy of any issue free, as long as the supply lasts; please request by volume and issue number from:

$$
\begin{aligned}
& \text { Agricultural Publications } \\
& \text { Room 22, Giannini Hall } \\
& \text { University of California } \\
& \text { Berkeley 4, California }
\end{aligned}
$$

The limit to nonresidents of California is 10 separate issues on a single order. A list of the issues still available will be sent on request. 


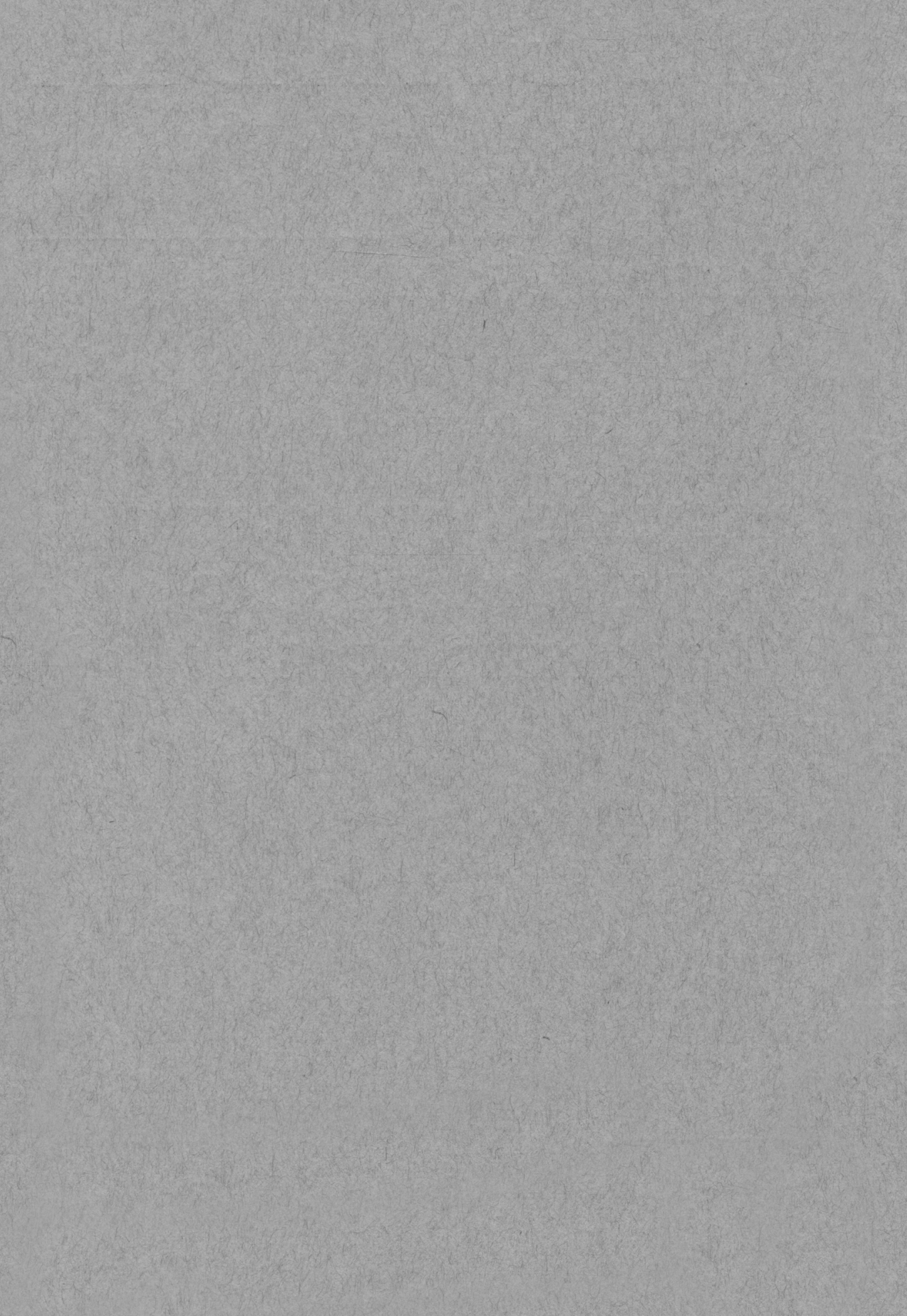

